\title{
Rare occurrences of non-cascading foreshock activity in Southern California
}

France.

This manuscript has been submitted for publication in Geophysical Research Letters. Please note that, despite having undergonepeer-review, the manuscript has yet to be formally accepted for publication. Subsequent versions of this manuscript may have slightly different content. If accepted, the final version of this manuscript will be available via the 'Peer-reviewed Publication DOI' link on the right-hand side of this webpage. Please feel free to contact any of the authors; we welcome feedback.

\section{Key Points:}

- We further investigate previous claims of significantly elevated seismic activity prior to large earthquakes in Southern California.

- 10 out of 53 mainshocks are preceded by anomalously high seismicity, but only 3 of these anomalies are exclusively related to the mainshock.

- These selected foreshock sequences are likely due to additional pre-slip, aseismic processes.

Corresponding author: Luc Moutote, lmoutote@unistra.fr 


\begin{abstract}
Earthquakes preceding large events are commonly referred to as foreshocks. They are often considered as precursory phenomena reflecting the nucleation process of the main rupture. Such foreshock sequences may also be explained by cascades of triggered events. Recent advances in earthquake detection motivates a reevaluation of seismicity variations prior to mainshocks. Based on a highly complete earthquake catalog, previous studies suggested that mainshocks in Southern California are often preceded by anomalously elevated seismicity. In this study, we test the same catalog against the Epidemic Type Aftershock Sequence model that accounts for temporal clustering due to earthquake interactions. We find that 10/53 mainshocks are preceded by a significantly elevated seismic activity compared with our model. This shows that anomalous foreshock activity are relatively uncommon when tested against a model of earthquake interactions. Accounting for the recurrence of anomalies over time, only 3/10 mainshocks present a mainshock-specific anomaly with a high predictive power.
\end{abstract}

\title{
Plain Language Summary
}

Recent observations in Southern California have suggested that the majority of large earthquakes are preceded by an elevated seismic activity. The anomalous character of those foreshock sequences is debated since episodes of elevated seismic activity are generally not followed by a mainshock. Here we compare these observations to a seismicity model that accounts for the natural clustering of seismicity due to earthquake interactions. Even using a highly complete earthquake catalog, we find that the majority of mainshocks present a seismic activity similar to what is expected by our model. We note that only 10 out of 53 selected mainshocks are preceded by episodes of anomalously high seismic activity. Whether these episodes cause the mainshock, or are simply coincident with it, is generally unclear: only for 3 out of these 10 instances the coincidence appears very unlikely. 


\section{Introduction}

Large earthquakes are often preceded by an increase in seismic activity, which is then referred to as a foreshock sequence (Jones \& Molnar, 1976; Bouchon et al., 2013; Marsan et al., 2014; Dodge et al., 1995, 1996; Reasenberg, 1999). Although these foreshock sequences are often referred to as precursors, a problem is the inherent difficulty to identify earthquakes as foreshocks before the mainshock occurs. In addition, we still do not fully understand the physical mechanisms that generate foreshocks and the reason why they occur. Two competing conceptual models have been proposed (Mignan, 2014). First, a "cascade model" where successive foreshock stress changes contribute to a slow cascade of random failures (possibly mediated by aseismic afterslip) ultimately leading to the mainshock (Helmstetter \& Sornette, 2003; Marzocchi \& Zhuang, 2011; Ellsworth \& Bulut, 2018). Second, a "slow pre-slip model" where foreshocks are passive tracers of an evolving fault loading process preceding the mainshock rupture (Dodge et al., 1996; Bouchon et al., 2011; Kato et al., 2016). The aseismic vs seismic contributions to the overall moment release during the precursory phase is ultimately what distinguishes these two models. Unfortunately, the aseismic part is generally difficult or merely impossible to estimate from the available observations, and one therefore needs to resort to indirect arguments, often pertaining to the spatial and temporal distribution of the foreshocks. Although recent observations of slow deformation transients lasting days to months before the mainshock favor the triggering of foreshocks by aseismic preslip (Socquet et al., 2017; Mavrommatis et al., 2014; Ito et al., 2013), the aseismic character of such precursory motion is vigorously debated (Ruiz et al., 2014; Bedford et al., 2015). In addition, foreshock sequences are not observed systematically before large earthquakes. However, this lack of systematic precursory observations might partly be due to the incompleteness of current seismicity catalogs (Mignan, 2014; Ross et al., 2019)

The southern California catalog was recently enhanced thanks to the template matching analysis conducted by Ross et al. (2019). The resulting QTM (Quake Template Matching) catalog includes more than 850,000 earthquakes (for the higher choice of threshold, see Section 2.1) in a 10 year-long period from 2008 to 2017 and is complete down to magnitudes near or below zero for the best resolved regions. Such a high degree of completeness of the QTM catalog motivates the evaluation of the statistical significance of seismic activity preceding large earthquakes in southern California. By comparing seismic activity before $M \geq 4$ earthquakes to a constant and local background rate, Trugman and Ross (2019, T\&R from here on) estimated that $72 \%$ of mainshocks in the QTM catalog are preceded by a significantly elevated seismic activity. With the same approach using the SCSN catalog, which includes less earthquakes, only $46 \%$ of mainshocks were detected with a significantly elevated seismic activity. These results suggest that detailed earthquake detections could bear important information about an impending earthquake. The seismic activity observed in the 20-day window before $M \geq 4$ earthquakes was later re-evaluated by van den Ende and Ampuero (2020, V\&A from here on) to investigate in which cases these increases in seismicity were significant compared to the natural fluctuations of the seismicity rate. In their approach, V\&A choose to test seismic activities smoothed at 20 days against a model that accounts for increases in seismicity. In this model, earthquake inter-event times (IETs) are drawn independently from a gamma distribution. This approach is motivated by the fact that IETs in seismic catalog tends to follow a gamma, rather than an exponential distribution (i.e. T\&R's background model) because the gamma distribution is more likely to fit the small IETs observed during clusters of earthquakes. Based on this analysis, V\&A estimated that only $33 \%$ of mainshocks are preceded in the last 20 days by a significantly elevated seismic activity, coming down to $18 \%$ when accounting for temporal fluctuations of such anomalies, i.e., anomalies taking place at random and therefore not specifically related to mainshock occurrences. 
For the sake of simplicity, we will now refer to as "foreshock activity" the seismic events observed in the 20 days immediately before $M \geq 4$ earthquakes. Although V\&A further addressed the significance of elevated foreshock activity in the QTM catalog, we believe that their analysis still underestimates the effect of earthquake clustering. Namely, the random sampling approach of V\&A assumes independent IETs, which is an over-simplification of the actual earthquake clustering observed during individual aftershock sequences. Indeed, during aftershock sequences, IETs are correlated rather than independent. We illustrate this concern in the supporting information (Text S4 and Figures S6) by applying the V\&A approach on synthetic ETAS catalogs. In this study, we consider that local earthquake interactions needs to be fully accounted for in order to identify foreshock activity that stands out from simple cascades of triggered seismicity.

We extend the studies of T\&R and V\&A by testing the statistical significance of elevated foreshock seismicity in the QTM catalog, accounting for local earthquake interactions. In this work, we use the temporal Epidemic Type Aftershock Sequences (ETAS) model, in which the seismicity rate at each time is represented by the superposition of a background rate and a rate linked to the aftershock triggering from past events (Ogata, 1988). This model is the simplest that can reproduce both the gamma distribution of IETs (Saichev \& Sornette, 2007) and their correlation during aftershock sequences. After selecting mainshocks using criteria similar to T\&R and V\&A, we extract ETAS parameters from the QTM catalog in the vicinity of each mainshock. We then compare the foreshock activity with ETAS predictions accounting for past seismicity. We find that the number of instances of anomalously elevated foreshock seismicity is significantly reduced when accounting for earthquake interactions (about $19 \%$ compared to $33 \%$ and $72 \%$ respectively in V\&A and T\&R). Moreover, out of these 10 cases, only 3 appear to be exclusively related to the subsequent occurrence of the mainshock.

\section{Data and methods}

\subsection{Mainshock selection}

We noticed that the full QTM catalog used by T\&R and V\&A suffers from episodic bursts of false detections, that occur due to too low a detection threshold (threshold fixed at 9.5 times the median absolute deviation (MAD) of the stacked correlation function). These bursts are easy to identify as they start or end at midnight, which is due to the MAD computation being performed over 24 hour long period starting at 00h00 UTC. To avoid any contamination of our analysis by such artifacts, we instead use the higher quality QTM catalog with a detection threshold at 12 times the MAD, for which these transients vanish or are strongly attenuated. In order to provide a fair comparison with previous results, we also present our analysis performed on the full catalog in the supporting information (Text S5 and Figures S7 and S8).

Using the higher quality QTM catalog, we then extract our own set of mainshocks with selection criteria similar to those used in T\&R: A mainshock must have magnitude $M \geq 4$, and must occur from 2009/01/01 to $2016 / 12 / 31$ within the geographic coordinates ranges $\left[32.68^{\circ} \mathrm{N}, 36.2^{\circ} \mathrm{N}\right]$ and $\left[118.80^{\circ} \mathrm{W}, 115.4^{\circ} \mathrm{W}\right]$. To be selected, a mainshock must be preceded by at least 10 earthquakes with no larger magnitude event in the year before and within a $20 \times 20 \mathrm{~km}^{2}$ horizontal box around its epicenter. 53 earthquakes were selected as mainshock according to these criteria. For each selected mainshock, we extract a 10-year long local catalog that includes all the seismicity observed within the $20 \times 20 \mathrm{~km}^{2}$ box with no depth cutoff.

We evaluate for each local catalog the local magnitude of completeness $M_{c}$ and remove all events with a magnitude $M<M_{c}$. We must acknowledge that removing all 
earthquakes of the QTM catalog below $M_{c}$ may remove potentially interesting features, but we consider that such features cannot be properly interpreted because they might reflect variation of the detection capability of the network and not real fluctuations of the seismicity rate. Therefore, to achieve a trade-off between completeness and retaining as many earthquakes as possible, we estimated manually the local $M_{c}$ as either the maximum of the local Gutenberg-Richter(G-R) frequency-magnitude distribution if this distribution decays smoothly for larger magnitudes, or the magnitude at which a notable break in slope is observed. Figure S1 of the supporting information shows the 53 local Gutenberg-Richter frequency-magnitude distributions and the corresponding estimated $M_{c}$ values.

\subsection{Inversion of ETAS parameters}

The ETAS model has two main ingredients: first, a background term which is time-independent and follows a Poisson process; second, a triggered term that depends on the past earthquake activity. The conditional intensity of the ETAS model (Ogata, 1988; Zhuang et al., 2012) is :

$$
\lambda(t)=\mu+\sum_{i \mid t_{i}<t} A \mathrm{e}^{\alpha\left(M_{i}-M_{c}\right)}\left(t-t_{i}+c\right)^{-p}
$$

where $\mu$ is the time-independent background seismicity rate. The sum in the right hand side of equation (1) describes the expected aftershock seismicity rate at time $t$ triggered by all previous events. $A$ and $\alpha$ are constant parameters describing respectively the global aftershock productivity of the region and the magnitude dependence in the number of triggered events. $M_{c}$ is the magnitude of completeness whereas $c$ and $p$ are the parameters of the Omori-Utsu law describing the time-decay in the aftershock seismicity rate. Therefore, in ETAS-like catalogs, temporally clustered seismicity only emerges from cascades of aftershocks.

For local catalogs associated with each mainshock, we fit the temporal ETAS model by maximizing a likelihood function with an Expectation - Maximization (EM) algorithm (Veen \& Schoenberg, 2008). We estimate parameters $A, c, p, \alpha$ and $\mu$ in equation (1) (all parameter values can be found in the supporting information). We run a first inversion where the ETAS parameters are constrained to be positive. We note that most $\alpha$ values are close to one. Larger $\alpha$ values are actually expected according to window-based methods (Helmstetter, 2005; Felzer et al., 2004), as well as following the argument that Bath's law, i.e., the fact that the difference in magnitude between the mainshock and its largest aftershock is independent of the mainshock's magnitude, requires that $\alpha=\beta=b \ln 10$ (Davidsen and Baiesi (2016) and references therein). Moreover, it has been shown that $\alpha$ estimates are particularly prone to model errors (e.g., Hainzl et al. $(2008,2013)$ ) and censoring effects (Sornette and Werner (2005); Seif et al. (2017)). Nandan et al. (2017) found that the $\alpha$ value is expected to vary between 1.7 and 2.2 when considering a larger portion of California and a longer period than the QTM catalog. A $\alpha$ value close to 2 may thus represent a more realistic value of the aftershock productivity for Californian earthquakes. Therefore, we perform a second inversion where we impose that $\alpha=2$. We thus obtain two sets of ETAS parameters (referred to as " $\alpha$ free" and " $\alpha=2$ " sets) to model the seismicity of local catalogs around each mainshocks. We also evaluate in the supporting information the sensitivity of our results to the uncertainty in ETAS estimates for both sets of parameters (cf., Text S3 and Figures S4-S5).

\subsection{Detection of seismicity anomalies based on the ETAS model}

We test the null hypothesis $H_{0}$ that the number of events observed in 20 days is smaller than or equal to the number of events predicted by the ETAS model for both sets of parameter estimates. If $H_{0}$ is rejected for both estimates, we assume that an 
anomalously high seismicity is detected in the window, suggesting that a mechanism other than simple ETAS cascading is required to explain the 20-day earthquake activity. The conditional intensity function in equation (1) allows to directly compute an expected seismicity rate at any time $t$ from the set of ETAS parameters $(A, c, p, \alpha$ and $\mu)$ and the knowledge of past seismicity $\left(t_{i}<t, M_{i}\right)$. By integrating this modelled seismicity rate, we can compute the expected number of earthquakes $\bar{N}$ in a time interval $T:$

$$
\bar{N}(t, T)=\int_{t-T}^{t} \lambda(u) \mathrm{d} u
$$

Here we set $T=20$ days similar to $T \& R$, which choice was also adopted by V\&A. We compute $\bar{N}$ over 20-day sliding windows, with a 1 day shift between two consecutive windows, and covering the full time range of the QTM catalog (i.e., 10 years). For all local catalogs around each mainshock, we then obtain two time-series of $\bar{N}$ generated using the two sets of inverted ETAS parameters $(\alpha$ free and $\alpha=2)$. Knowing $\bar{N}$, the probability of actually observing $N_{\text {obs }}$ earthquakes in a given 20-day time-interval is given by the Poisson distribution with mean $\bar{N}$ :

$$
P\left(N_{o b s}\right)=\frac{\bar{N}^{N_{o b s}} \mathrm{e}^{-\bar{N}}}{N_{o b s} !}
$$

We then define the probability of observing at least $N_{o b s}$ events over 20 days for the null hypothesis as:

$$
p=P\left(N \geq N_{o b s}\right)=1-\sum_{n=0}^{N_{o b s}-1} \frac{\bar{N}^{n} \mathrm{e}^{-\bar{N}}}{n !}
$$

Following $\mathrm{T} \& \mathrm{R}$ and $\mathrm{V} \& \mathrm{~A}$, we use the probability threshold $p<0.01$ to reject the hypothesis $H_{0}$ that $N_{o b s}$ is in agreement with the expected number of events $\bar{N}$. A small $p$-value would therefore correspond to anomalously elevated seismicity rate compared with ETAS predictions.

\section{Results}

The detection of seismicity rate anomalies in a 20-day sliding window is illustrated in Figure 1 for the seismicity located in the vicinity of 4 mainshocks. For each mainshock, the top subplot shows the time-evolution of p-values measured for the two sets of ETAS parameters ( $\alpha$ free and $\alpha=2$ ) while the bottom subplot shows the observed seismicity (i.e., magnitude vs time). For the two examples on top (Mainshock IDs 10832573 and 37301704), we notice that the 20-day foreshock activity is consistent with ETAS predictions with a p-value above 0.01 in the last 20-days window prior to the mainshock. In these cases, our null hypothesis $H_{0}$ cannot be rejected with a confidence of 99\%. The two examples on the bottom (Mainshock IDs 14898996 and 37299263) show p-values that are below 0.01 before the mainshock for both ETAS estimates. In these cases, the observed foreshock seismicity is higher than the expected ETAS cascading seismicity with a confidence level of at least $99 \%$.

In total, we find that 10 out of 53 mainshocks are preceded by an anomalously high 20-day activity with respect to ETAS predictions. Therefore, these mainshocks are likely preceded by complementary aseismic processes other than cascades of aftershocks. However, this result must be taken in perspective with the overall ability of the ETAS models to explain fluctuations in seismicity rates over the entire catalog. As pointed out by V\&A, the predictive power of an anomalously high foreshock activity is reduced if seismicity anomalies are frequently detected without being followed by a large event. The significance of an anomalously high foreshock activity being predictive of future large events should therefore be assessed given the overall ability 
a) (ID)10832573 - 2010/11/04 19:39
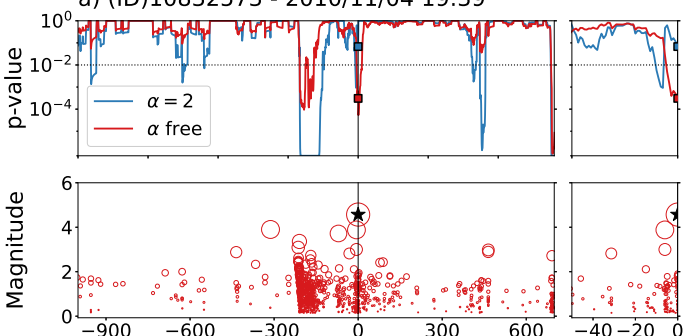

b) (ID) $14898996-2010 / 12 / 1519: 16$
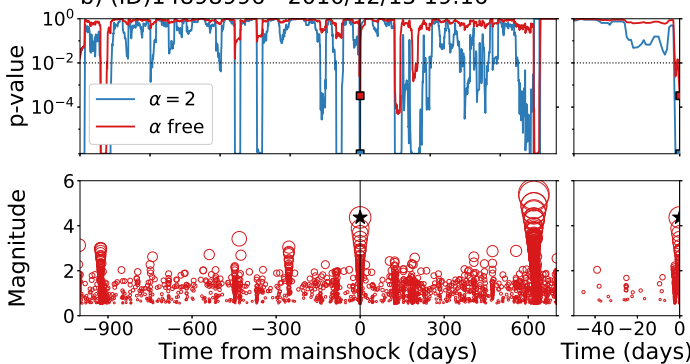

C) (ID)37301704 - 2015/01/04 03:18

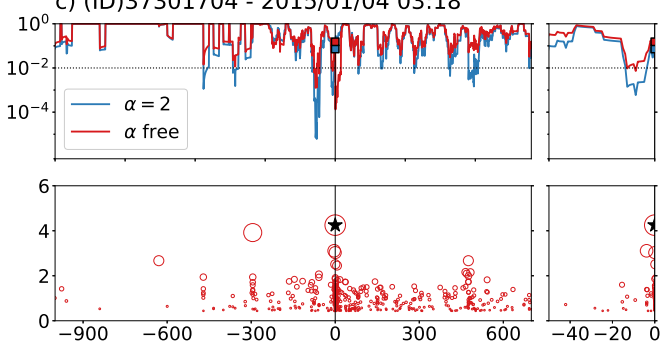

d) (ID)37299263 - 2016/01/24 15:32
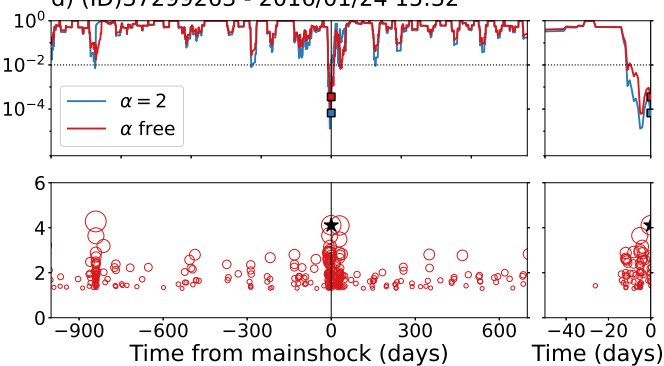

Figure 1. The 20-day sliding window analysis for 4 examples of mainshocks (black star at $t=0$ ) and their local catalog. Mainshocks IDs are (a) 10832573, (b) 37301704, (c) 14898996 and (d) 37299263. (Top graphs) probability $p$ that ETAS explains the observed seismicity, computed for the two sets of ETAS parameters $\alpha=2$ and $\alpha$ free. The p-value for the last 20-day window prior to the mainshock is shown with a thick square. The significance threshold of $p=0.01$ is shown with the horizontal dotted line. (Bottom graphs) magnitude vs time for the local catalogs in the $20 \times 20 \mathrm{~km}^{2}$ box around each mainshock. The right inset is a zoom around the foreshock window.

of ETAS predictions to explain the seismicity in the vicinity of the mainshock. For example, in the case of mainshock ID 14898996 in Figure 1c, ETAS predictions are unable to explain the observed seismicity at several occasions during the course of the catalog. Our null hypothesis $H_{0}$ is thus rejected for numerous 20-day windows with p-values smaller than the p-value of the foreshock window. On the other hand, Figure 1d shows that mainshock 37299263 presents an anomalously high seismicity rate almost exclusively in the 20 days preceding the mainshock. Such an elevated seismicity rate is thus highly correlated with the mainshock occurrence. We believe that the uniqueness of the anomaly observed before mainshock ID 37299263 is more likely to evidence predictive non-cascading mechanisms than mainshock ID 14898996.

Therefore, to quantify the significance of detected foreshock anomalies, we compare p-values in the foreshock window with the distribution of p-values over the entire 10-year catalog. For each mainshock, an anomalous foreshock activity is considered mainshock-specific if $\hat{p}$, the proportion of 10-year p-values lower or equal than the foreshock p-value, is less than $1 \%$. This arbitrary threshold of $1 \%$ allows to discriminate between catalogs with frequent anomalous activities and those with foreshock activities that correspond to the strongest anomalies of their region. This is summarized in Figure 2b. Using such temporal specificity criterion, we identify that 7 out of the 10 anomalous foreshock activity already mentioned occur in regions with recurrent seismicity anomalies stronger than the foreshock one. Therefore, we argue that only 3 out of 53 mainshocks present a clear mainshock-specific anomalous activity. We note that this final selection is highly dependent on the choice of the $\hat{p}$ threshold. Figure $2 \mathrm{~b}$ shows that all 10 selected sequences present less than $10 \%$ of 20 -day windows over 
10-years below the foreshock window p-value. The final selection of 3 out of 53 mainshock is therefore more like a refined selection of mainshocks with a local seismicity that best fit ETAS with a notable exception during foreshock time ranges.

We complement this analysis by declustering the local catalogs. The probability $\omega_{i}$ that earthquake $i$ is a background earthquake is defined as $\omega_{i}=\frac{\mu}{\lambda\left(t_{i}\right)}$, and can be calculated once the ETAS parameters are estimated. We then simply count the numbers of background earthquakes as the sums of $\omega_{i}$ in 20 day long windows. We denote $N_{0}$ this count for the last 20 days prior to the mainshock, and by $N$ all the counts for all the time windows before the mainshock (not just the last one). Following the same rationale that stimulated our previous analysis, we first compare $N_{0}$ to the Poisson distribution with a mean $\bar{N}$ equal to the mean of $N$, select the mainshocks for which $P\left(>N_{0} \mid \bar{N}\right)<0.01$ for the two sets of ETAS parameters (1st test), and finally check whether these selected sequences display other anomalously strong bursts of background earthquakes by computing the probability that $N$ can be greater than $N_{0}$ (2nd test). We finally select those short-listed mainshocks for which the latter probability is less than 0.01 (again, for the two sets of ETAS parameters). Figure 3 shows the results of this declustering approach. Only mainshocks 14598228 and 14600292 are preceded by an anomalously high foreshock activity (1st test) according to this declustering approach. According to our 2nd test, these two anomalies are also specific to the subsequent mainshock occurrences (i.e., p-value $\leq 0.01$ ). These two foreshock sequences were also identified in our previous approach based on the predicted number of events according to the ETAS model. The difference in results between the declustering approach and the former method is due to the fact that declustering only leaves a small number of background earthquakes, and therefore has a strong tendency to significantly lower the p-values.

\section{Discussion}

We use the highly complete QTM catalog of Ross et al. (2019) for southern California to further investigate the significance of anomalous high foreshock activity previously reported by T\&R and V\&A. As mentioned before, those studies did not fully address whether the temporal clustering of earthquakes observed during aftershock sequences is a possible explanation for the observed elevated foreshock activities. This clustering is considered as one of the possible origins of the high seismic activity observed before large earthquakes (Helmstetter \& Sornette, 2003; Marzocchi \& Zhuang, 2011; Ellsworth \& Bulut, 2018). In practice, small $M<4$ earthquakes trigger small aftershock sequences during which a larger $M>4$ event is more likely to occur than at more quiet times. In this regard, high activity preceding a mainshock can naturally stem from such earthquake interactions and cascading without necessarily requiring an external pre-slip phenomenon. To address this concern, we use the ETAS model to discriminate which instances of QTM foreshock activities exhibit higher seismicity rates than expected from earthquake interactions.

We first assess the probability $p$ that a given 20-day foreshock sequence can be explained by ETAS earthquake clustering. Using $p<0.01$ as a threshold, our results indicate that $\sim 19 \%$ (10 out of 53 ) of mainshocks are preceded by increases in seismicity higher than $99 \%$ of the earthquake rates predicted by ETAS. The 20-day temporal evolution of these 10 anomalous foreshock sequences is detailed in Text S2 and Figure S9. In a second step, we further distinguish 3 out these 10 cases as being specific to the subsequent mainshock, i.e., the chance to see such a significant increase of activity occurring at random is less than $1 \%$. The anomalously high seismicity of these 3 foreshock sequences is thus highly correlated with the $M \geq 4$ mainshock occurrences and likely to be controlled by aseismic nucleation processes. We notice that this number (3 out of 10 ) would raise to 5 if accepting a threshold at $1.5 \%$ rather than $1 \%$, cf. Figure $2 \mathrm{~b}$. The complementary declustering approach restricts the anomalously high 
a) Receiver operating characteristic curve (ROC)

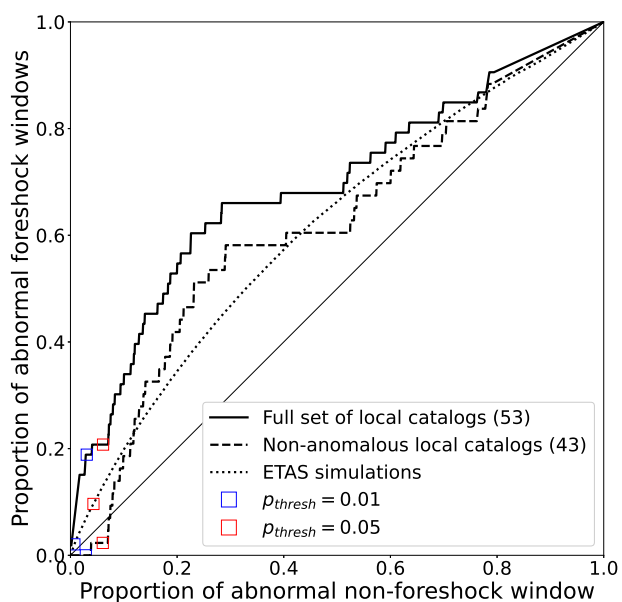

b) Among all 20-day windows, proportion of p-values below the foreshock p-value

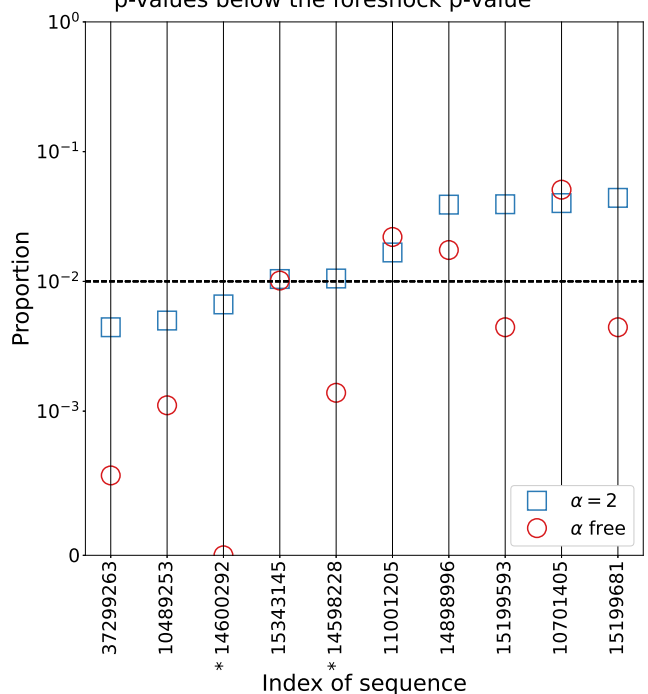

Figure 2. (a) Receiver Operating Characteristic (ROC) curves for our detection of anomalous foreshock windows. For a varying threshold p-value $p_{\text {thresh }}$, the curves show the proportion of foreshock windows below $p_{\text {thresh }}$ against the proportion of non-foreshock windows below $p_{\text {thresh }}$. ROC curves are drawn for both the full set of 53 local catalogs and the set of 43 catalogs left after removing the 10 anomalous sequences of section 3 (with $p<0.01$ ). We also include the ROC curve corresponding to the average of 53 sets of 1000 ETAS simulations computed using the $\alpha$ free ETAS parameters obtained in section 2.2. Note that ETAS simulations display a curved ROC, the departure from the "no-gain" line being particularly clear when considering large $p_{\text {thresh }}$ values. This departure is weak for $p_{\text {thresh }} \leq 0.01$, with a gain of about 2 at maximum $\left(p_{\text {thresh }}=0.01\right)$. (b) Proportion $\hat{p}$ of windows with a p-value lower or equal to the 20-day foreshock window p-value, among all 20-day windows over 10 years. The proportion $\hat{p}$ is shown here for the 10 anomalously high foreshock activity and for the two ETAS estimates. We consider an anomalously high foreshock activity to be specifically related to a mainshock if $\hat{p}$ is below 0.01 for both ETAS estimates. Here, we identify 3 foreshock anomalies that are specific to subsequent mainshocks for both sets of ETAS parameters. Note that $\hat{p}$ is significantly sensitive to the value of $\alpha$. Labels preceded by a star are mainshock IDs of the two anomalously high foreshock activity detected with the declustering approach.

foreshock activity to only two mainshock-specific sequences. A possible over-estimation of the background rate can be a cause for this more conservative selection. Even if the definitions of an anomalously elevated seismicity differ, Mainshock IDs related to the anomalously high foreshock activities detected in T\&R, V\&A and this study can be found in Table S1 of the supporting information. The Southern Californian location of these sequences are also compared in Figure S10.

We must emphasize that these results, along with those of T\&R and V\&A, likely depend on the initial choice of focusing on foreshocks in a 20 day period prior to each mainshock. Using a longer or shorter time-window may therefore provide different results. Moreover, the fixed $20 \times 20 \mathrm{~km}^{2}$ horizontal spatial window used in this study implies that all events in this box are evaluated with the same weight. This can artificially enhance the triggering role of foreshocks that are relatively far from the 

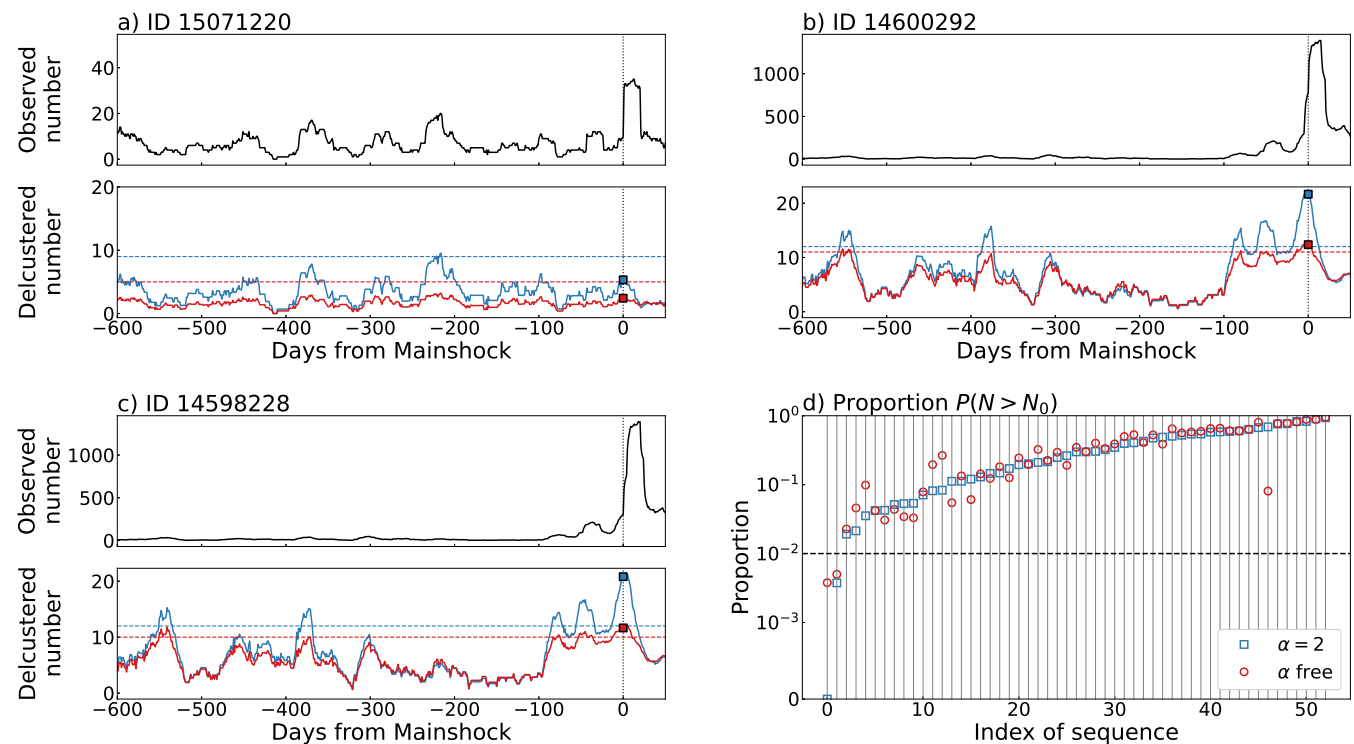

Figure 3. (a,b,c) Number of earthquakes in 20 day long windows counting (top) all earthquakes and (bottom) background earthquakes only, for 3 selected mainshocks. The number for the last window prior to the mainshock is shown with a thick square. The dashed lines show, for the two sets of ETAS parameters (free $\alpha$ in red, $\alpha=2$ in blue) the limit over which the Poisson probability becomes less than 0.01. (d) Probability $P\left(N>N_{0}\right)$ that the last 20 days are anomalously active compared to the past, for the two sets of ETAS parameters; the sequence is selected as a mainshock-specific anomalous activity after declustering if this probability is less than 0.01 (2nd test) and if $N_{0}$ is above the dashed line (1st test). Mainshocks 14598228 and 14600292 correspond to indices 0 and 1 on this graph, and are the only mainshocks with both probabilities less than 0.01. All indices can be linked with their mainshock ID thanks to Table S2.

mainshock. The ETAS model used here would need to be extended to a space-time model in order to exploit the distance between earthquakes and to help to discriminate such cases (Zhuang et al., 2011, for a review). While this development does not appear over complicated, and was already investigated in Seif et al. (2019), the addition of several model parameters and the use of an isotropic spatial kernel for which no clear consensus exists (Moradpour et al., 2014) is likely to undermine the robustness and significance of the resuts.

The exact number of detected foreshock anomalies obviously depends on the significance threshold that we have fixed to $p<0.01$ following T\&R and V\&A. To assess the impact of this arbitrary choice, we evaluate how the proportion of detected anomalous high foreshock activity changes as a function of the $\mathrm{p}$-value threshold $p_{\text {thresh }}$. This result is compared with the proportion of windows that have $p<p_{\text {thresh }}$ without being followed by a mainshock (i.e., false positives). We thus compute the Receiver Operating Characteristic (ROC) curve as shown in Figure 2a. If the occurrence of anomalously elevated activity was not a sign of an incoming mainshock, then the ROC curve would follow a 1 to 1 straight line (hereafter referred to as the no-gain line). We find that there is positive correlation between preceding high activity and mainshock occurrence: the information gain is measured by the ratio of true positives over false positives, which is practically constant and close to 6 for $p_{\text {thresh }} \leq 0.05$. We however notice that significant departure from this no-gain line also exists in ETAS simulations computed with the same 53 sets of parameters as obtained for the local catalogs. Figure 2 shows that a 
large $p_{\text {thresh }}$ (i.e., $p_{\text {thresh }}>0.01$ ) allows to detect anomalous foreshock activities (i.e., a positive gain) in ETAS simulations, even though there is by definition no pre-slip in this model. This is caused by the clustering properties of the model: in the rare occasions where the observed number of earthquakes $N_{o b s}$ in a window largely exceeds the expected number $\bar{N}$, then the occurrence of earthquakes immediately after this window is more likely, including the occurrence of a mainshock. As an effect, the ROC curve departs from the no-gain line. We however notice that there is no information gain on the magnitude of the forthcoming earthquakes, as expected. We conclude that choosing too large a value of $p_{\text {thresh }}$ may lead to the detection of "foreshock cascades" prior to mainshocks, which are not related to aseismic processes (e.g., preslip). According to our simulations, $p_{\text {thresh }}=0.01$ appears as an acceptable threshold to discriminate a cascading-like seismicity from other processes that would also enhance the seismic activity: at $p_{\text {thresh }}=0.01$, the information gain for ETAS is about 2, compared to about 6 for the observed seismicity (cf., $p_{\text {thresh }}=0.01$ in Figure 2 ). This additional gain is mostly controlled by the 10 sequences we found to be anomalous: quite obviously, removing them from the calculations implies that the ROC curve is equal to zero at $p_{\text {thresh }}=0.01$. Therefore, these 10 anomalous foreshock sequences suggest the existence of a precursory pattern before some $M \geq 4$ earthquakes stronger than expected from ETAS simulations.

Our results strengthen previous reports that earthquake activity precursory to mainshocks can sometimes deviate from simple clustering properties (as modelled by ETAS; Lippiello et al., 2019; Seif et al., 2019). Our approach is however different. For example, compared to Seif et al. (2019), we seek to explain the last 20 days prior to mainshocks knowing all past seismicity (including activity in the last 20 days), by comparing what number of earthquakes would be "normally" expected (in the sense of ETAS) to the observed number. In contrast, Seif et al. (2019) compared observations to the number of foreshocks predicted by ETAS simulations not constrained by past seismicity. Our method is indeed close to the residual analyses of Ogata $(1988,1989$, 1992) and Ogata et al. (2003), which is here performed individually on a set of 53 mainshocks thanks to the improved completeness of the QTM dataset.

\section{Conclusions}

According to our analyses, the low magnitude of completeness of the QTM catalog does not warrant the detection of aseismically-driven foreshock sequences in the 20-days window preceding isolated mainshocks. More than $80 \%$ of mainshocks are preceded in the last 20 days by activity exhibiting seismicity rates that are consistent with ETAS predicted rates, even when the magnitude of completeness is as low as $M_{c}=0$. For these cases, earthquake interactions and local stress changes are a good candidate to explain all observed increases in seismicity rates prior to the mainshock. We find 10 mainshocks that are preceded in the last 20 days by a significantly high seismic activity. These cases show seismic activity that significantly differ from ETAS cascades, and are thus likely controlled by aseismic processes. Among those 10 cases, we distinguish 3 cases that exhibit non-ETAS like seismicity that is very likely specifically related to the mainshock; these 3 cases are the best evidences of a possible nucleation phase.

High quality earthquake datasets complete to low magnitudes are in any case required to pursue and develop efforts for understanding when and where aseismic pre-slip can lead to a large shock. Foreshocks remain the best observable to study preparatory processes, if they exist (Nakatani, 2020). First, increasing the location accuracy and the number of small earthquakes substantially improves the statistical significance of any test conducted to assess the reality of pre-slip processes, when comparing to the cascade (null) hypothesis. Second, the availability of large datasets allows to increase the number of potential mainshocks to be analyzed, hence offering 
more robust conclusions. Finally, we suggest that pre-slip seismicity analysis should be evaluated along other near-fault observables (such as GPS data (Socquet et al., 2017), strainmeter data (Roeloffs, 2006), variations in groundwater level or flow rate (Roeloffs, 1988), radon emission rate (Ghosh et al., 2009), changes in seismic velocities as imaged by pairwise seismic station cross-correlation functions (von Seggern \& Anderson, 2017) ) whenever available, to independently assess any possible aseismic mechanisms at work during the preparation of large earthquakes.

\section{Acknowledgments}

This study is based on the QTM seismicity catalog accessible via the Southern California Earthquake Data Center (https://scedc.caltech.edu/data/qtm-catalog.html). This project has received funding from the European Research Council (ERC, under the European Union's Horizon 2020 research and innovation program under grant agreement No. 805256). We thank the editors, Victor Tsai and Germán Prieto, along with the reviewers, Andy Michael and Peter Shearer, for their comments which helped improve the manuscript.

\section{References}

Bedford, J., Moreno, M., Schurr, B., Bartsch, M., \& Oncken, O. (2015). Investigating the final seismic swarm before the Iquique-Pisagua $2014 \mathrm{Mw} 8.1$ by comparison of continuous gps and seismic foreshock data. Geophysical Research Letters, 42(10), 3820-3828.

Bouchon, M., Durand, V., Marsan, D., Karabulut, H., \& Schmittbuhl, J. (2013). The long precursory phase of most large interplate earthquakes. Nature Geoscience, 6(4), 299-302. doi: 10.1038/ngeo1770

Bouchon, M., Karabulut, H., Aktar, M., Ozalaybey, S., Schmittbuhl, J., \& Bouin, M.-P. (2011). Extended nucleation of the $1999 \mathrm{Mw} 7.6$ Izmit earthquake. Science, 331(6019), 877-880. doi: 10.1126/science.1197341

Davidsen, J., \& Baiesi, M. (2016). Self-similar aftershock rates. Physical Review E, 94 (2), 022314. doi: 10.1103/PhysRevE.94.022314

Dodge, D. A., Beroza, G. C., \& Ellsworth, W. L. (1995). Foreshock sequence of the 1992 Landers, California, earthquake and its implications for earthquake nucleation. Journal of Geophysical Research: Solid Earth, 100(B6), 9865-9880. doi: 10.1029/95JB00871

Dodge, D. A., Beroza, G. C., \& Ellsworth, W. L. (1996). Detailed observations of California foreshock sequences: Implications for the earthquake initiation process. Journal of Geophysical Research: Solid Earth, 101(B10), 22371-22392. doi: 10.1029/96JB02269

Ellsworth, W. L., \& Bulut, F. (2018). Nucleation of the 1999 Izmit earthquake by a triggered cascade of foreshocks. Nature Geoscience, 11(7), 531-535. doi: 10 .1038/s41561-018-0145-1

Felzer, K. R., Abercrombie, R. E., \& Ekström, G. (2004). A common origin for aftershocks, foreshocks, and multiplets. Bulletin of the Seismological Society of America, 94 (1), 88-98. doi: 10.1785/0120030069

Ghosh, D., Deb, A., \& Sengupta, R. (2009). Anomalous radon emission as precursor of earthquake. Journal of Applied Geophysics, 69(2), 67-81. doi: 10.1016/ j.jappgeo.2009.06.001

Hainzl, S., Christophersen, A., \& Enescu, B. (2008). Impact of earthquake rupture extensions on parameter estimations of point-process models. Bulletin of the Seismological Society of America, 98(4), 2066-2072. doi: 10.1785/0120070256

Hainzl, S., Zakharova, O., \& Marsan, D. (2013). Impact of aseismic transients on the estimation of aftershock productivity parameters. Bulletin of the Seismological Society of America, 103(3), 1723-1732. doi: 10.1785/0120120247 
Helmstetter, A. (2005). Importance of small earthquakes for stress transfers and earthquake triggering. Journal of Geophysical Research, 110(B5), B05S08. doi: 10.1029/2004JB003286

Helmstetter, A., \& Sornette, D. (2003). Foreshocks explained by cascades of triggered seismicity. Journal of Geophysical Research, 108(B10). doi: 10.1029/ 2003JB002409

Ito, Y., Hino, R., Kido, M., Fujimoto, H., Osada, Y., Inazu, D., ... Ashi, J. (2013). Episodic slow slip events in the Japan subduction zone before the 2011 Tohoku-Oki earthquake. Tectonophysics, 600, 14-26.

Jones, L., \& Molnar, P. (1976). Frequency of foreshocks. Nature, 262(5570), 677679.

Kato, A., Fukuda, J., Kumazawa, T., \& Nakagawa, S. (2016). Accelerated nucleation of the 2014 Iquique, Chile Mw 8.2 earthquake. Scientific Reports, 6(1), 24792. doi: $10.1038 /$ srep24792

Lippiello, E., Godano, C., \& de Arcangelis, L. (2019). The relevance of foreshocks in earthquake triggering: A statistical study. Entropy, 21(2), 173. doi: 10.3390/ e21020173

Marsan, D., Helmstetter, A., Bouchon, M., \& Dublanchet, P. (2014). Foreshock activity related to enhanced aftershock production. Geophysical Research Letters, 41(19), 6652-6658.

Marzocchi, W., \& Zhuang, J. (2011). Statistics between mainshocks and foreshocks in Italy and Southern California. Geophysical Research Letters, 38(9).

Mavrommatis, A. P., Segall, P., \& Johnson, K. M. (2014). A decadal-scale deformation transient prior to the $2011 \mathrm{Mw} 9.0$ Tohoku-oki earthquake. Geophysical Research Letters, 41(13), 4486-4494. doi: 10.1002/2014GL060139

Mignan, A. (2014). The debate on the prognostic value of earthquake foreshocks: A meta-analysis. Scientific Reports, 4(1), 4099.

Moradpour, J., Hainzl, S., \& Davidsen, J. (2014). Nontrivial decay of aftershock density with distance in Southern California. Journal of Geophysical Research: Solid Earth, 119(7), 5518-5535. doi: https://doi.org/10.1002/2014JB010940

Nakatani, M. (2020). Evaluation of phenomena preceding earthquakes and earthquake predictability. Journal of Disaster Research, 15(2), 112-143. doi: 10 .20965/jdr.2020.p0112

Nandan, S., Ouillon, G., Wiemer, S., \& Sornette, D. (2017). Objective estimation of spatially variable parameters of epidemic type aftershock sequence model: Application to California. Journal of Geophysical Research: Solid Earth, 122(7), 5118-5143. doi: 10.1002/2016JB013266

Ogata, Y. (1988). Statistical models for earthquake occurrences and residual analysis for point processes. Journal of the American Statistical Association, 83(401), 9-27. doi: 10.1080/01621459.1988.10478560

Ogata, Y. (1989). Statistical model for standard seismicity and detection of anomalies by residual analysis. Tectonophysics, 169(1-3), 159-174. doi: 10.1016/0040 $-1951(89) 90191-1$

Ogata, Y. (1992). Detection of precursory relative quiescence before great earthquakes through a statistical model. Journal of Geophysical Research, 97(B13), 19845. doi: 10.1029/92JB00708

Ogata, Y., Katsura, K., \& Tanemura, M. (2003). Modelling heterogeneous spacetime occurrences of earthquakes and its residual analysis. Journal of the Royal Statistical Society: Series C (Applied Statistics), 52(4), 499-509. doi: 10.1111/ 1467-9876.00420

Reasenberg, P. A. (1999). Foreshock occurrence before large earthquakes. Journal of Geophysical Research: Solid Earth, 104(B3), 4755-4768. doi: https://doi.org/ 10.1029/1998JB900089

Roeloffs, E. A. (1988). Hydrologic precursors to earthquakes: A review. Pure and Applied Geophysics, 126(2-4), 177-209. doi: 10.1007/BF00878996 
Roeloffs, E. A. (2006). Evidence for aseismic deformation rate changes prior to earthquakes. Annual Review of Earth and Planetary Sciences, 34(1), 591-627. doi: 10.1146/annurev.earth.34.031405.124947

Ross, Z. E., Trugman, D. T., Hauksson, E., \& Shearer, P. M. (2019). Searching for hidden earthquakes in Southern California. Science, 364(6442), 767-771. doi: $10.1126 /$ science.aaw6888

Ruiz, S., Metois, M., Fuenzalida, A., Ruiz, J., Leyton, F., Grandin, R., .. Campos, J. (2014). Intense foreshocks and a slow slip event preceded the 2014 Iquique Mw 8.1 earthquake. Science, 345(6201), 1165-1169.

Saichev, A., \& Sornette, D. (2007). Theory of earthquake recurrence times. Journal of Geophysical Research, 112(B4). doi: 10.1029/2006JB004536

Seif, S., Mignan, A., Zechar, J. D., Werner, M. J., \& Wiemer, S. $\quad$ (2017). Estimating ETAS: The effects of truncation, missing data, and model assumptions. Journal of Geophysical Research: Solid Earth, 122(1), 449-469. doi: 10.1002/2016JB012809

Seif, S., Zechar, J. D., Mignan, A., Nandan, S., \& Wiemer, S. (2019). Foreshocks and their potential deviation from general seismicity. Bulletin of the Seismological Society of America, 109(1), 1-18. doi: 10.1785/0120170188

Socquet, A., Valdes, J. P., Jara, J., Cotton, F., Walpersdorf, A., Cotte, N., .. Norabuena, E. (2017). An 8 month slow slip event triggers progressive nucleation of the 2014 Chile megathrust. Geophysical Research Letters, 44 (9), 4046-4053. doi: 10.1002/2017GL073023

Sornette, D., \& Werner, M. J. (2005). Apparent clustering and apparent background earthquakes biased by undetected seismicity. Journal of Geophysical Research, 110(B9). doi: 10.1029/2005JB003621

Trugman, D. T., \& Ross, Z. E. (2019). Pervasive foreshock activity across Southern California. Geophysical Research Letters, 46(15), 8772-8781. doi: 10.1029/ 2019GL083725

van den Ende, M. P. A., \& Ampuero, J. (2020). On the statistical significance of foreshock sequences in Southern California. Geophysical Research Letters, $47(3)$. doi: 10.1029/2019GL086224

Veen, A., \& Schoenberg, F. P. (2008). Estimation of space-time branching process models in seismology using an EM-type algorithm. Journal of the American Statistical Association, 103(482), 614-624. doi: 10.1198/016214508000000148

von Seggern, D. H., \& Anderson, J. G. (2017). Velocity change in the zone of a moderate $\mathrm{Mw} 5.0$ earthquake revealed by autocorrelations of ambient noise and by event spectra. Pure and Applied Geophysics, 174(5), 1923-1935. doi: 10.1007/s00024-017-1521-2

Zhuang, J., Harte, D., Werner, M. J., Hainzl, S., \& Zhou, S. (2012). Basic models of seismicity: Temporal models. Community Online Resource for Statistical Seismicity Analysis, Theme $V(1)$.

Zhuang, J., Werner, M. J., Hainzl, S., Harte, D., \& Zhou, S. (2011). Basic models of seismicity: Spatiotemporal models. Community Online Resource for Statistical Seismicity Analysis, Theme V(1), 20. 


\section{Supporting Information for "Rare occurrences of non-cascading foreshock activity in Southern California"}

L. Moutote ${ }^{1}$, D. Marsan ${ }^{2}$, O. Lengliné ${ }^{1}$, Z. Duputel ${ }^{1}$

${ }^{1}$ Institut Terre et Environnement de Strasbourg, UMR7063, Université de Strasbourg/EOST, CNRS, Strasbourg, France.

${ }^{2}$ Institut des Sciences de la Terre, UMR5275, Université Savoie Mont Blanc, CNRS, Le Bourget du Lac, France.

Pre-print Warning

This manuscript has been submitted for publication in Geophysical Research Letters. Please note that, despite having undergonepeer-review, the manuscript has yet to be formally accepted for publication. Subsequent versions of this manuscript may have slightly different content. If accepted, the final version of this manuscript will be available via the 'Peer-reviewed Publication DOI' link on the right-hand side of this webpage. Please feel free to contact any of the authors; we welcome feedback.

February 16, 2021, 12:43pm 
$\mathrm{X}-2$

\section{Contents of this file}

1. Text S1 to S5

2. Figures $\mathrm{S} 1$ to $\mathrm{S} 10$

3. Tables $\mathrm{S} 1$ to $\mathrm{S} 2$

\section{Introduction}

\section{Text S1: Overview of the p-values results for the 53 local catalogs}

To evaluate the overall ability of the ETAS model to reproduce the observed 20-day seismicity and to isolate catalogs with an anomalously high foreshock activity, we computed p-values distribution over each entire local catalog (with a 20-day sliding window) and for the two ETAS parameters estimates. The 10-year p-value distributions of each selected local catalog are presented in Figure S2 (in red for $\alpha$ free and in blue for $\alpha=2$ ). Square dots indicate the p-value observed in the foreshock window.

We use a probability threshold of 0.01 for both ETAS estimates to reject our nullhypothesis $H_{0}$ that 20-day foreshock window seismicity can be explained by an ETAS seismicty. We find that 10 out of the 53 mainshocks selected in this study present an anomalously high foreshock activity.

Text S2: The 10 anomalously high 20-day foreshock clusters

February 16, 2021, 12:43pm 
Figure S9 shows the 10 anomalously high 20-day foreshock clusters detected in this study. We note that the 10 related mainshocks occur at different times but mainly in the South-Est of southern California. The foreshock activity is not really consistent between mainshocks but seems to follow 3 main spatio-temporal patterns, either: (1) a group of foreshocks less than $1 \mathrm{~km}$ away from the future mainshock position and homogeneous over the 20-day window (IDs: 14599228, 37299263, 11001205); (2) a sudden burst occurring just before the mainshock time and a few $\mathrm{km}$ from the mainshock position (IDs: 15199593, 14898996, 10489253, 15343145); (3) three mainshocks occur isolated by a few $\mathrm{km}$ from their foreshock locations (ID: 10701405, 14600292, 15199681). We note that 2 out of the 10 mainshocks with anomalous foreshock sequences occur close and less than 20 days after one of the 8 remaining "anomalous" mainshocks. As a consequence, the related 20-day windows are interlaced and may evidence similar anomalous activities. For example, the foreshock sequence related to Mainshock ID 14600292 occurs almost at the same location as Mainshock ID 14599228 but 4 days later. We note that the two successive mainshocks respect our mainshock selection criterion since $M_{14600292}>M_{14599228}>4$. The foreshock sequence of ID 14600292 is interlaced with the foreshock and aftershock activity of previous Mainshock ID 14599228. As a consequence, we observe seismic activity mainly clustered at the Mainshock ID 14599228 location, $2 \mathrm{~km}$ away from Mainshock ID 14600292. Even if these two mainshocks are studied independently in our approach, they both occur following the same burst of foreshock activity that therefore led to the production of two large magnitude events. Mainshocks ID 15199593 and 15199681 follow the same conclusions.

February 16, 2021, 12:43pm 


\section{Text S3: P-value sensitivity to uncertainty on ETAS estimates}

We evaluate the ETAS estimates uncertainties obtained with the ExpectationMaximization algorithm for a few local catalogs to understand their influence on p-value results. For computational efficiency, we have only selected 14 mainshocks to perform the uncertainty analysis. This selection include 12 mainshocks with the lowest foreshock p-values (see Figure S2) and the 2 remaining mainshocks presented in Figure 1 of the main text. Note that we discarded Mainshock ID 37374687 because its local catalog is very large, making it very computionnally expensive to run this Monte-Carlo approach. For each selected mainshock, we compute the ETAS estimates uncertainties as follow:

1. We generate between 100 and 200 10-year long synthetic ETAS catalogues using the initial sets of ETAS estimates (i.e. 200 simulations with the $\alpha=2$ set and 200 simulations with the $\alpha$ free set).

2. We re-estimate new sets of ETAS parameters for each simulation with the Expectation-Maximization algorithm. Note that the 200 simulations computed with $\alpha=2$ are re-inverted with the $\alpha=2$ constrain. We thus obtain two distributions of synthetic ETAS estimates representing the initial ETAS estimate uncertainties.

3. We use each new synthetic ETAS estimate to compute the p-value curve for a sliding 20-day window. These p-values are therefore based on the actual QTM local catalogs but using the ETAS parameters deduced from the synthetic catalogs: we obtain twice 200 p-values for each time window, allowing us to infer uncertainties on the p-values.

February 16, 2021, 12:43pm 
The uncertainties of ETAS parameter estimates from 200 simulations are shown in Figure S3 for mainshock ID 37299263. The distribution is Gaussian shaped, centered around the initial value and with a moderate standard deviation. Foreshock window p-values computed with ETAS uncertainties are displayed in Figure S5 for the 14 selected mainshocks. Figure 1 of the main text is reproduced in Figure S4 with the corresponding foreshock p-value uncertainties. We note that the p-value sensitivity is moderate and does not change the selection of anomalously high foreshock activity when considering the 0.01 threshold.

\section{Text S4: V\&A approach with synthetic ETAS catalogs}

In this section, we illustrate how the V\&A approach behaves on aftershock sequences by applying it to synthetic realizations of a temporal ETAS seismicity model (cf., Figure S6). Synthetic ETAS catalogs are able to reproduce a temporally clustered seismicity. In such model, clustering activity emerges spontaneously from random cascades of aftershocks. This is illustrated in Figure S6a with observable aftershock sequences initially triggered by several $M \sim 3$ events and a $M=4$ earthquake. By construction, such a synthetic catalog does not contain any foreshock activity other than that due to earthquake interactions. As for natural seismicity, the distribution of inter-event times (IETs) of an ETAS catalog tends to a gamma distribution (cf., FigureS6b). Following V\&A, if we independently resample the IETs of Figure S6b, we obtain for instance the catalog shown in Figure S6c in which the temporal clustering disappeared (even if IETs have the same distribution by construction). In particular, there is no visible aftershock sequences 
$\mathrm{X}-6$

following $M \sim 3$ events contrary to catalog observations. To further quantify the limitations of such a random sampling approach, we generate 1000 realizations of 5-years duration synthetic ETAS catalogs and extract $M \geq 4$ mainshocks as in section 2.1 of the main article. Following V\&A, we then sample a Probability Mass Function (PMF) of the expect number of event in 20 day windows assuming independent gamma realization of IETs (Figure S6d). We extract the probability $p$ that independent IETs can explain foreshock seismicity by confronting this PMF with the "observed" number of events in the 20 days prior synthetic mainshocks (Figure S6e). Assuming the same significance threshold of $p<0.01$ as in T\&R and V\&A, Figure S6e shows that more than $10 \%$ of mainshocks are preceded by an anomalously high seismic activity even though they are actually explained by cascades of aftershocks. The 1000 synthetic ETAS catalogs are also tested against the second approach of V\&A. In this approach, the PMF is sampled empirically by counting the number of events in 20-days windows randomly distributed over the $[-380,-20[$ period with respect to the mainshock origin time (FigureS6d). As for independent IETs sampled from a gamma distribution, the empirical approach of V\&A shows that more than $10 \%$ of mainshocks are preceded by an anomalously high earthquake activity (FigureS6f). Therefore, the two approaches of V\&A struggle to properly consider causal earthquakes interactions and their corresponding seismicity rate increases.

\section{Text S5: Reproducing the ETAS analysis on Trugman and Ross (2019) main- shock selection over the QTM 9.5 dev catalog}

February 16, 2021, 12:43pm 
The Quake Template Matching catalog of Southern California provided by Ross, Trugman, Hauksson, and Shearer (2019) is presented as two separate catalogs with different confidence levels on the detection of events. The full QTM catalog (i.e. "QTM 9.5 dev" : detection threshold at 9.5 times the median absolute deviation (MAD) of the stacked correlation function) is used for foreshock analysis by Trugman and Ross (2019) and van den Ende and Ampuero (2020). We noticed that QTM 9.5 dev suffers from episodic bursts of false detections, that occur due to too low a threshold. To avoid any contamination of our analysis by such artifacts, we instead use the higher quality QTM catalog with a detection threshold at 12 times the MAD (i.e. QTM $12.5 \mathrm{dev}$ ), for which these transients vanish or are strongly attenuated. The use of the QTM 12.5 dev catalog implies that the mainshock selection is slightly different from the one used by T\&R and V\&A.

In order to provide a fair comparison with the results of $T \& R$ and $V \& A$, we show in Figure S7 our ETAS analysis performed on the QTM 9.5 dev for the T\&R mainshock selection (46 events). Apart from the mainshock selection, the method used is the same as the one presented in the main article.

Using the same criteria for the selection of anomalous high foreshock activity, we find that 9 out of 46 (20\%) foreshock windows are anomalous. Only 2/46 of these anomalously high foreshock activity ( $5 \%$ ) are considered mainshock specific when considering the 10year variations of anomalies (Figure S8). We note that Mainshock IDs 37299263 and 14600292 are found as having mainshock-specific anomalous activity for both of QTM catalogs and mainshock selection criteria. Figure S10 summarizes the location of the 
$\mathrm{X}-8$

detected anomalously high foreshock activity for the analysis mentioned in this study (T\&R, V\&A, ETAS QTM 9.5 dev and QTM $12.5 \mathrm{dev})$.

\section{References}

Ross, Z. E., Trugman, D. T., Hauksson, E., \& Shearer, P. M. (2019). Searching for hidden earthquakes in Southern California. Science, 364(6442), 767-771. doi: 10.1126/ science.aaw6888

Trugman, D. T., \& Ross, Z. E. (2019). Pervasive foreshock activity across Southern California. Geophysical Research Letters, 46(15), 8772-8781. doi: 10.1029/ 2019GL083725

van den Ende, M. P. A., \& Ampuero, J. (2020). On the statistical significance of foreshock sequences in Southern California. Geophysical Research Letters, $47(3)$. doi: 10.1029/2019GL086224

February 16, 2021, 12:43pm 
Table S1. QTM anomalous foreshock sequences

\begin{tabular}{|c|c|c|c|}
\hline Approach & $\begin{array}{l}\text { Mainshock } \\
\text { selection }\end{array}$ & $\begin{array}{c}\text { Anomalous high } \\
\text { foreshock activity }(p<0.01)\end{array}$ & $\begin{array}{l}\text { Mainshock specific } \\
\text { anomalous activity }\end{array}$ \\
\hline${ }^{a}{ }^{a}$ Poisson & $\begin{array}{c}\text { T\&R } \\
(\mathrm{N}=46)\end{array}$ & $\begin{array}{l}14383980,15200401,37374687 \\
15481673,15296281,15520985 \\
10370141,11413954,10527789 \\
15476961,37507576,15475329 \\
37510616,14898996,11373458 \\
14571828,37301704,11001205 \\
14600292,37298672,10321561 \\
15507801,11006189,10489253 \\
37299263,15014900,14403732 \\
37166079,14406304,37644544 \\
15153497,15267105,37243591\end{array}$ & $\overline{\mathrm{NA}}$ \\
\hline${ }^{b} \mathrm{Gamma}$ & $\begin{array}{c}\mathrm{T} \& \mathrm{R} \\
(\mathrm{N}=46)\end{array}$ & $\begin{array}{l}15200401,15481673,10527789 \\
37510616,14898996,11373458 \\
37301704,11001205,14600292 \\
11006189,10489253,37299263 \\
15071220,14406304,15267105\end{array}$ & NA \\
\hline${ }^{b}$ Empirical & $\begin{array}{c}\text { T\&R } \\
(\mathrm{N}=46)\end{array}$ & $\begin{array}{c}15200401,10527789,14898996 \\
37301704,11001205,14600292 \\
11006189,10489253,37299263 \\
14406304\end{array}$ & $\mathrm{NA}$ \\
\hline $\begin{array}{c}{ }^{c} \text { ETAS } \\
\text { Expected } \bar{N}\end{array}$ & $\begin{array}{c}\mathrm{T} \& \mathrm{R} \\
(\mathrm{N}=46)\end{array}$ & $\begin{array}{l}15071220,10527789,14406304 \\
15507801,14898996,10489253 \\
14600292,37299263,11001205\end{array}$ & 14600292,37299263 \\
\hline $\begin{array}{c}{ }^{c} \text { ETAS } \\
\text { Expected } \bar{N}\end{array}$ & $\begin{array}{l}\text { This study } \\
(\mathrm{N}=53)\end{array}$ & $\begin{array}{c}37299263,10489253,14600292 \\
15343145,14598228,11001205 \\
14898996,15199593,10701405 \\
15199681\end{array}$ & $37299263,10489253,14600292$ \\
\hline $\begin{array}{c}{ }^{c} \text { ETAS } \\
\text { Declustering }\end{array}$ & $\begin{array}{c}\mathrm{T} \& \mathrm{R} \\
(\mathrm{N}=46)\end{array}$ & $\begin{array}{c}10321561,14600292,15296281 \\
37374687\end{array}$ & 14600292 \\
\hline $\begin{array}{c}{ }^{c} \text { ETAS } \\
\text { Declustering }\end{array}$ & $\begin{array}{l}\text { This study } \\
(\mathrm{N}=53)\end{array}$ & 14598228,14600292 & 14598228,14600292 \\
\hline
\end{tabular}


Table S2. [Uploaded separately] This study' mainshock selection in the QTM 12.5 dev Southern Californian catalog and their respective two set of ETAS inverted parameters $(A, c, p, \alpha, \mu)$ for $\alpha$ free or $\alpha=2$ (Each mainshock is related to a local catalog defined as all the seismicity within a 20 by $20 \mathrm{~km}^{2}$ box around the mainshock and above the local magnitude of completeness $M_{c}$ ). For each ETAS parameter estimates we present the 20-day foreshock window p-value evaluated with the ETAS expected 20-day seismicity and the declustering approach.

February 16, 2021, 12:43pm 
Count
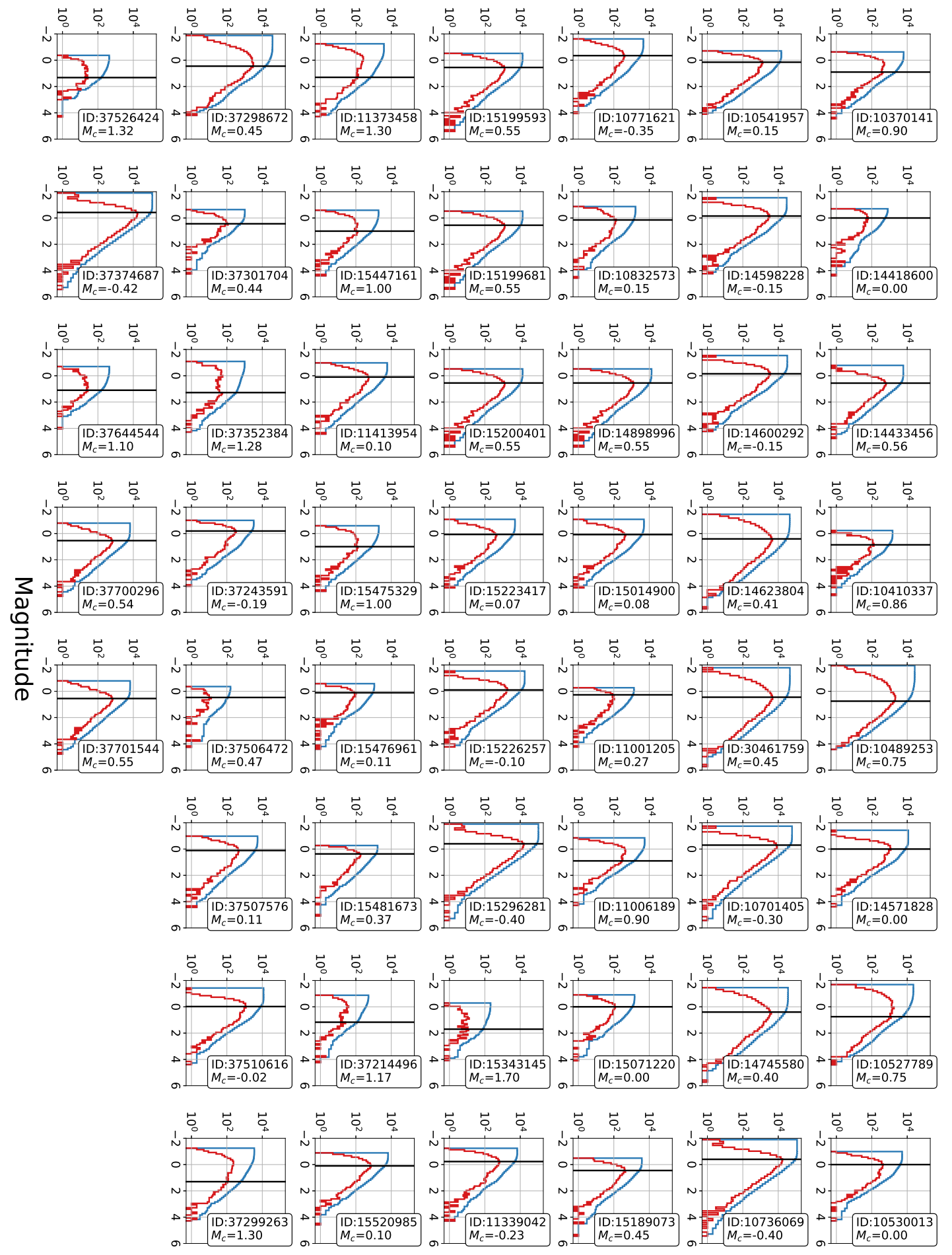

Figure S1. The magnitude of completeness $M_{c}$ of local catalogs observed in a 20 by 20 $\mathrm{km}^{2}$ box around the 53 mainshocks as selected in this study. (red) The frequency-magnitude distribution. (blue) The corresponding cumulative distribution. (black) The estimated magnitude of completeness.

February 16, 2021, 12:43pm 


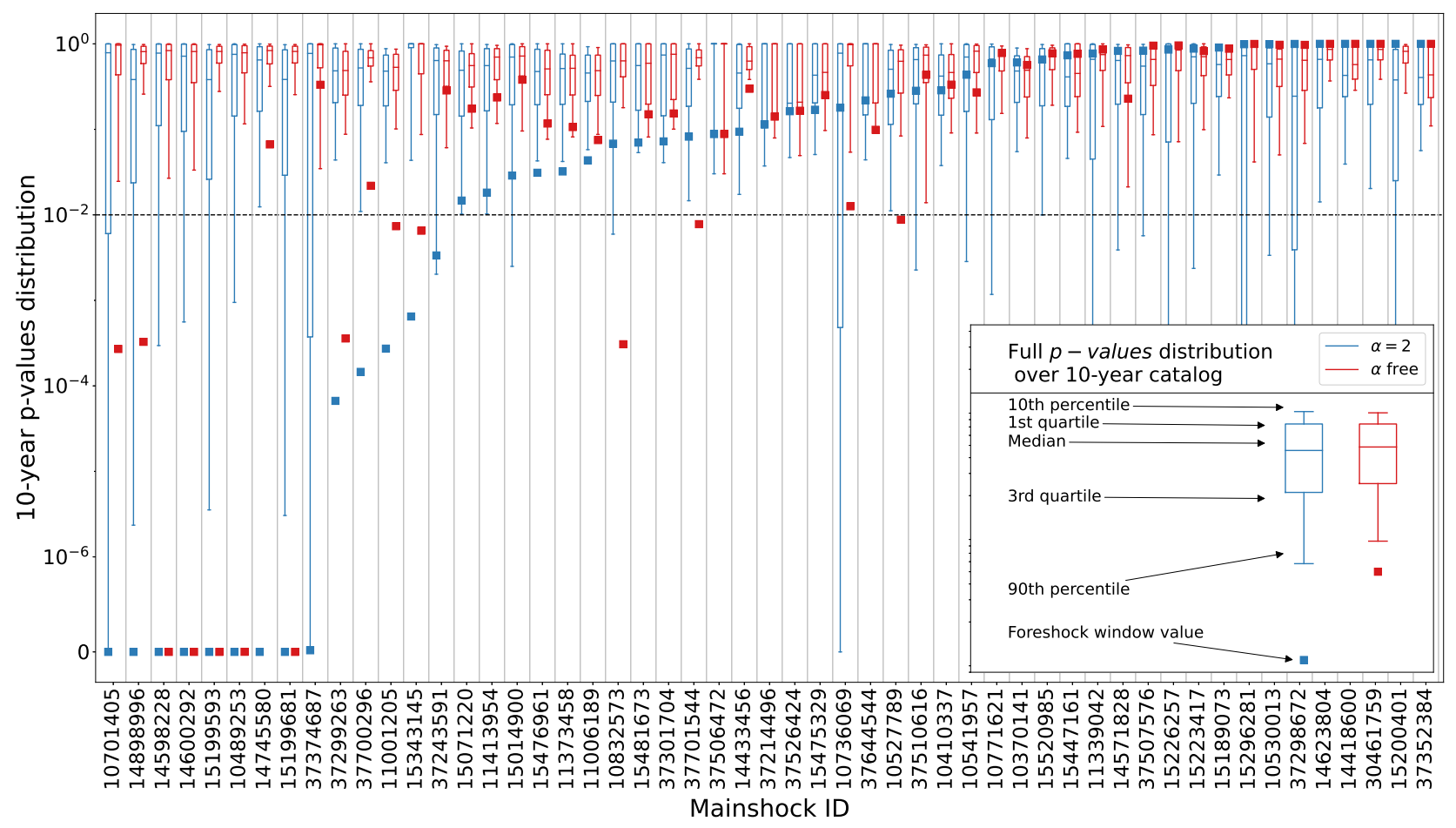

Figure S2. [QTM $12.5 \mathrm{dev}]$ ETAS expected 20-day seismicity analysis over our mainshock selection from the QTM 12.5 dev catalog. For each selected mainshock, the boxplots give the p-value distribution computed with a 20-day sliding window over the 10-year for the two sets of ETAS parameter estimates. The squared dot is the p-value computed for the 20day foreshock window. The black dashed line is the $0.01 \mathrm{p}$-value threshold. A foreshock window p-value is anomalous if it is below the threshold for both sets of ETAS parameter estimates. We here find 10 (among 46) anomalous foreshock windows.

February 16, 2021, 12:43pm 
a) (ID) 37299263: ETAS estimates from simulations with $\alpha$ free
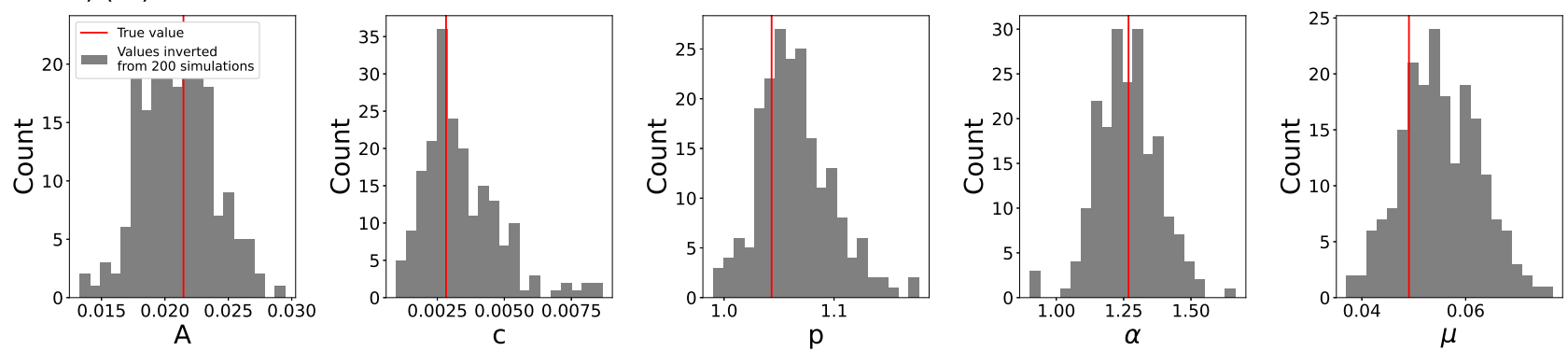

b) (ID) 37299263: ETAS estimates from simulations with $\alpha=2$
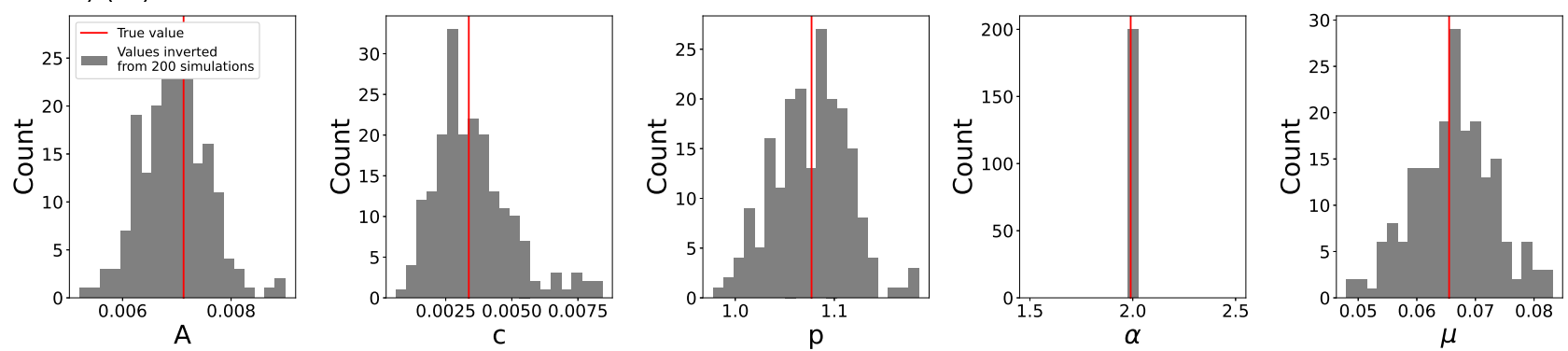

Figure S3. ETAS estimate uncertainties inverted from 200 synthetic ETAS catalogs, along with the 'real' ETAS estimates for the local catalog of mainshock ID 37299263. a) Uncertainties from 200 simulations computed with $\alpha$ free and re-inverted with no constrains on $\alpha$. b) Uncertainties from 200 simulations computed with $\alpha=2$ and re-inverted with $\alpha$ fixed to 2 .

February 16, 2021, 12:43pm 

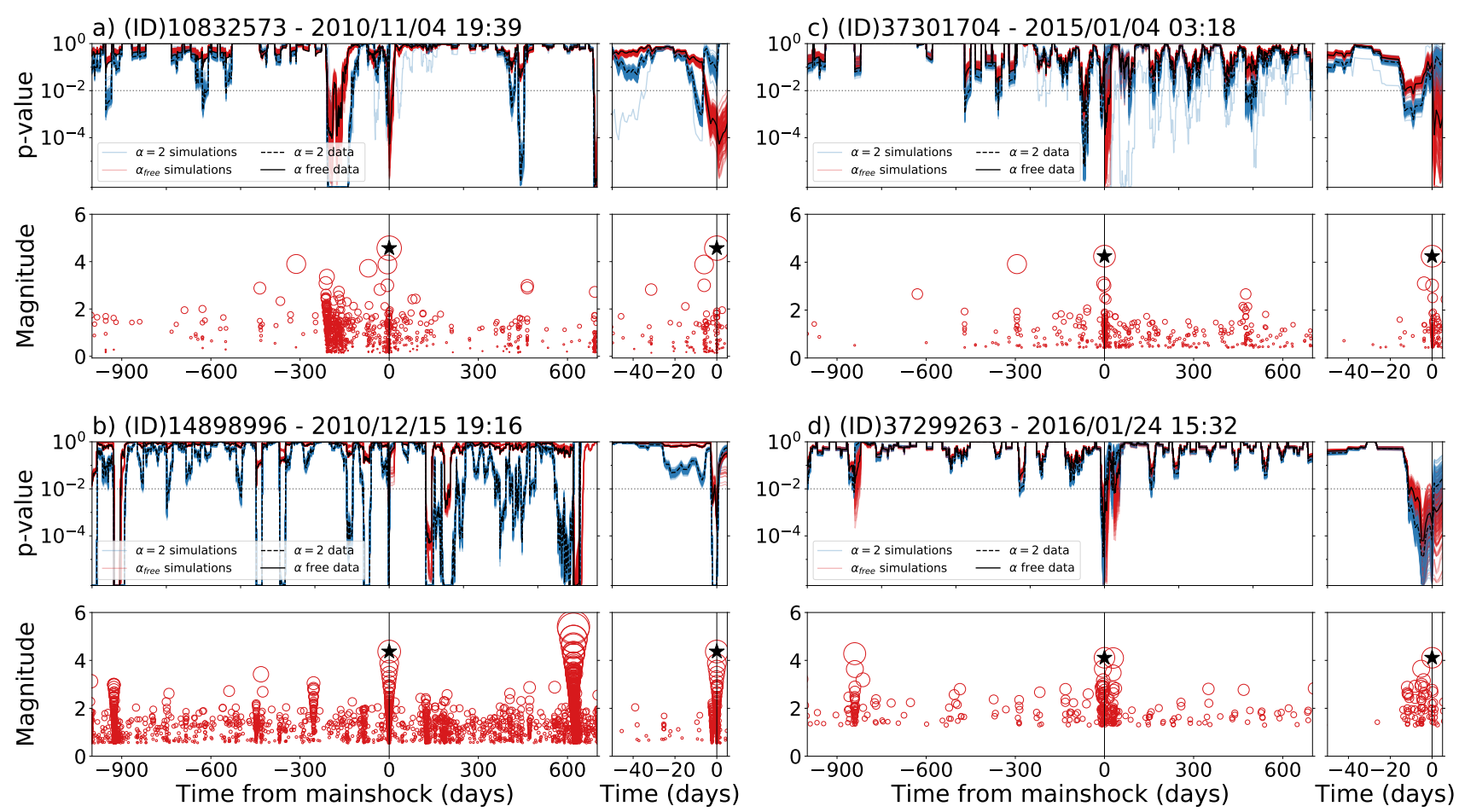

Figure S4. As in Figure 1 of the main text but including uncertainties: The 20-day sliding window analysis for 4 mainshocks (black star at $t=0$ ) and their local catalogs. (Top graphs) probability $p$ that ETAS explains the observed seismicity, computed for the two sets of ETAS estimates inverted from the data (i.e. ' $\alpha=2$ data' and ' $\alpha$ free data') and their uncertainties computed from simulations (i.e. ' $\alpha=2$ simulations' and ' $\alpha$ simulations'). The significance threshold of $p=0.01$ is shown with the horizontal dotted line. (Bottom graphs) magnitude vs time for the local catalogs in the $20 \times 20 \mathrm{~km}^{2}$ box around each mainshock. The right inset is a zoom around the foreshock window. 


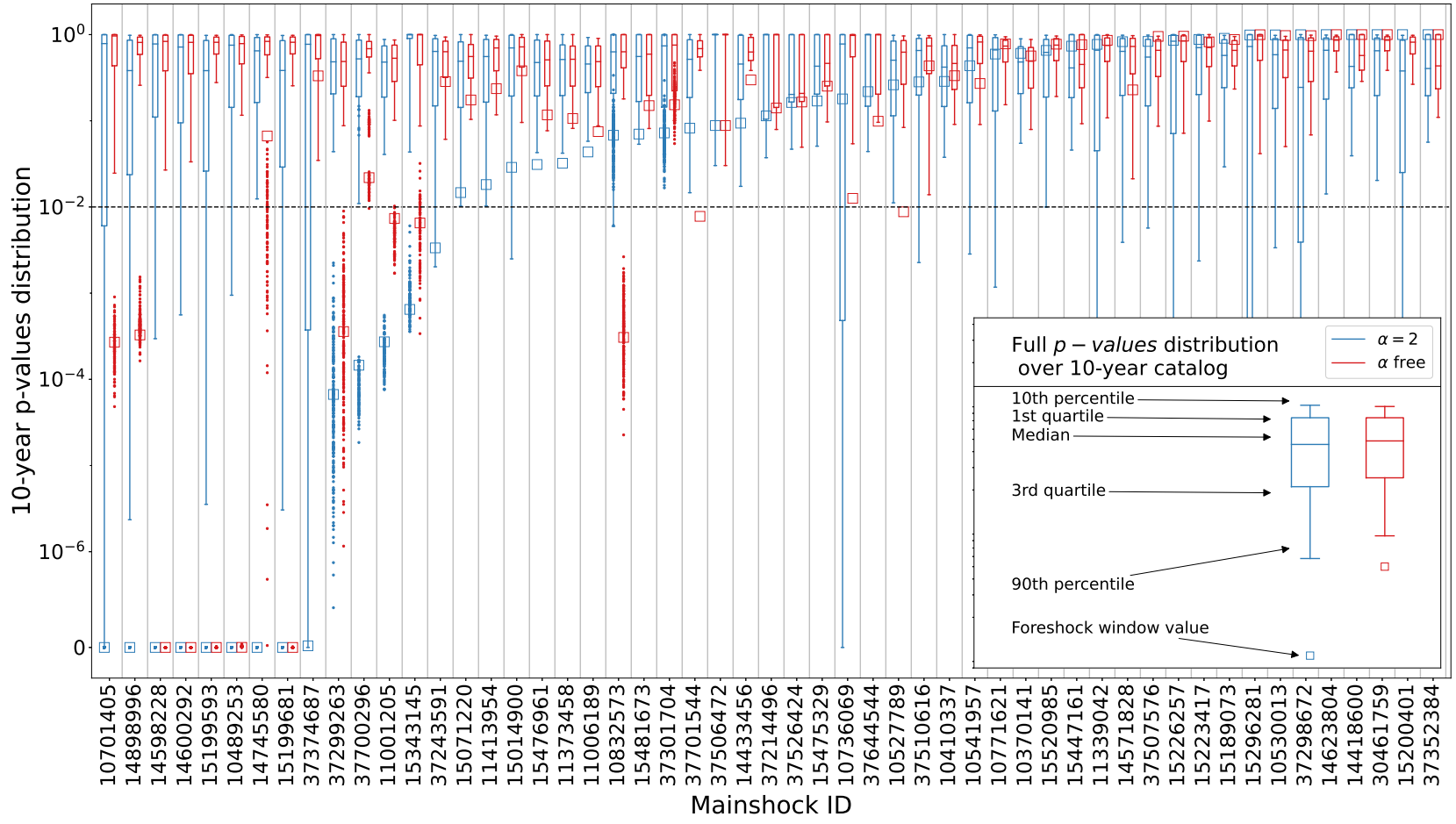

Figure S5. As in Figure S2 but including the p-value distribution obtained with our ETAS estimate uncertainties. Each dot is a foreshock p-value computed with one the set of ETAS parameter estimates inverted from the simulations.

February 16, 2021, 12:43pm 
a) Synthetic ETAS catalog

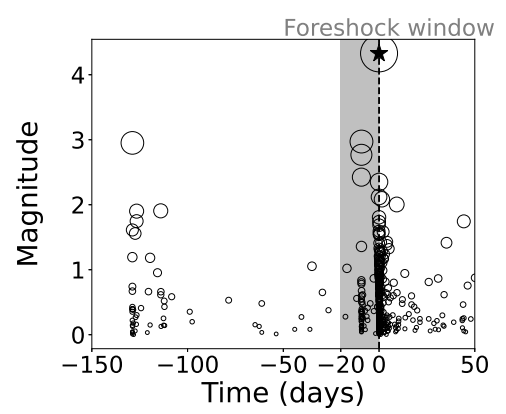

d) Number of events over 20 days

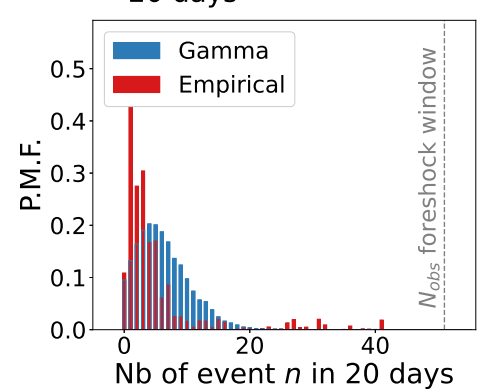

b) Distribution of IETS

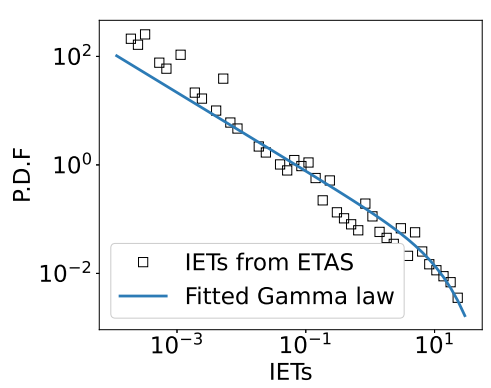

e) p-values from

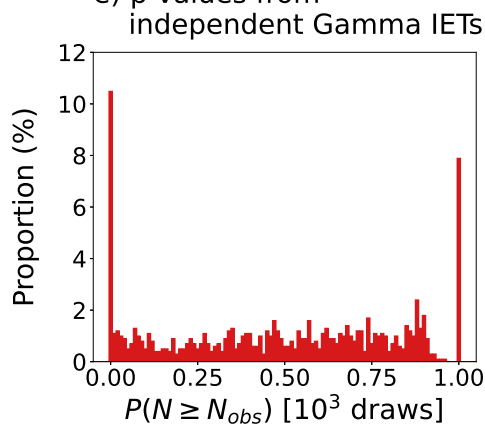

c) Catalog from re-shuffled IETs

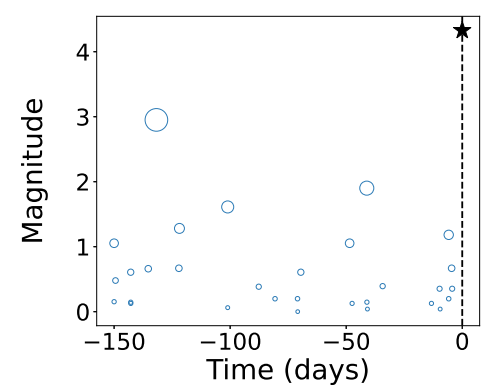

f) p-values from the

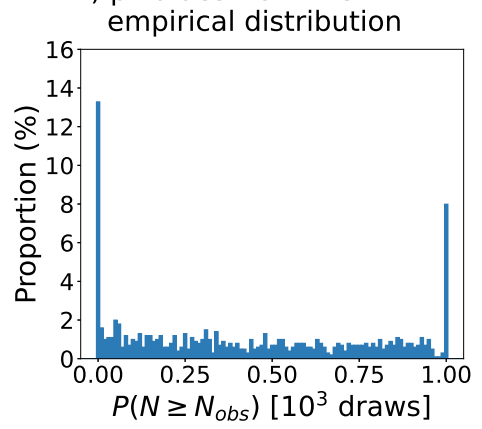

Figure S6. (a) A realisation of a synthetic ETAS catalogue $\left(\alpha=2, p=1.1, c=10^{-3}, \mu=0.1\right.$, $\beta=2.23$ corresponding to a $b$-value of 1 for the Gutenberg-Richter law, $\left.M_{c}=0\right)$ and its 20-day foreshock window as defined by van den Ende and Ampuero (2020). The $M \geq 4$ is considered here as the mainshock. (b) IETs distribution of this ETAS catalogue observed in the $[-380,-20[$ window and its fitted gamma law. (c) IETs reshuffling of the $[-380,-20[$ days window. Note that clustered events are no longer related to the distribution of magnitude. (d) The sampled gamma/empirical probability mass functions (PMFs) of the number of events expected in the 20day window according to the two approaches of V\&A. The red vertical dashed line corresponds to the number of events $N_{\text {obs }}$ actually observed in the ETAS 20-day foreshock window. Distribution of the foreshock probability $p=P\left(N \geq N_{o b s}\right)$ using V\&A first approach (drawing of independent, gamma-distributed IETs), for the 1000 synthetic ETAS catalogs. (f) Same as (e) but for the V\&A second (empirical) approach (counting the number of earthquakes within random 20 day windows included in the $[-380,-20[$ period before the mainshock). More than $10 \%$ of the ETAS foreshock windows are detected with an anomalous seismicity $(p<0.01)$ although no anomaly is actually present. In (e) and (f), the p-value spike at 1 correspond to windows with $N_{o b s}=0$ or $N_{o b s}$ far from the minimum of the gamma/empirical PMF 


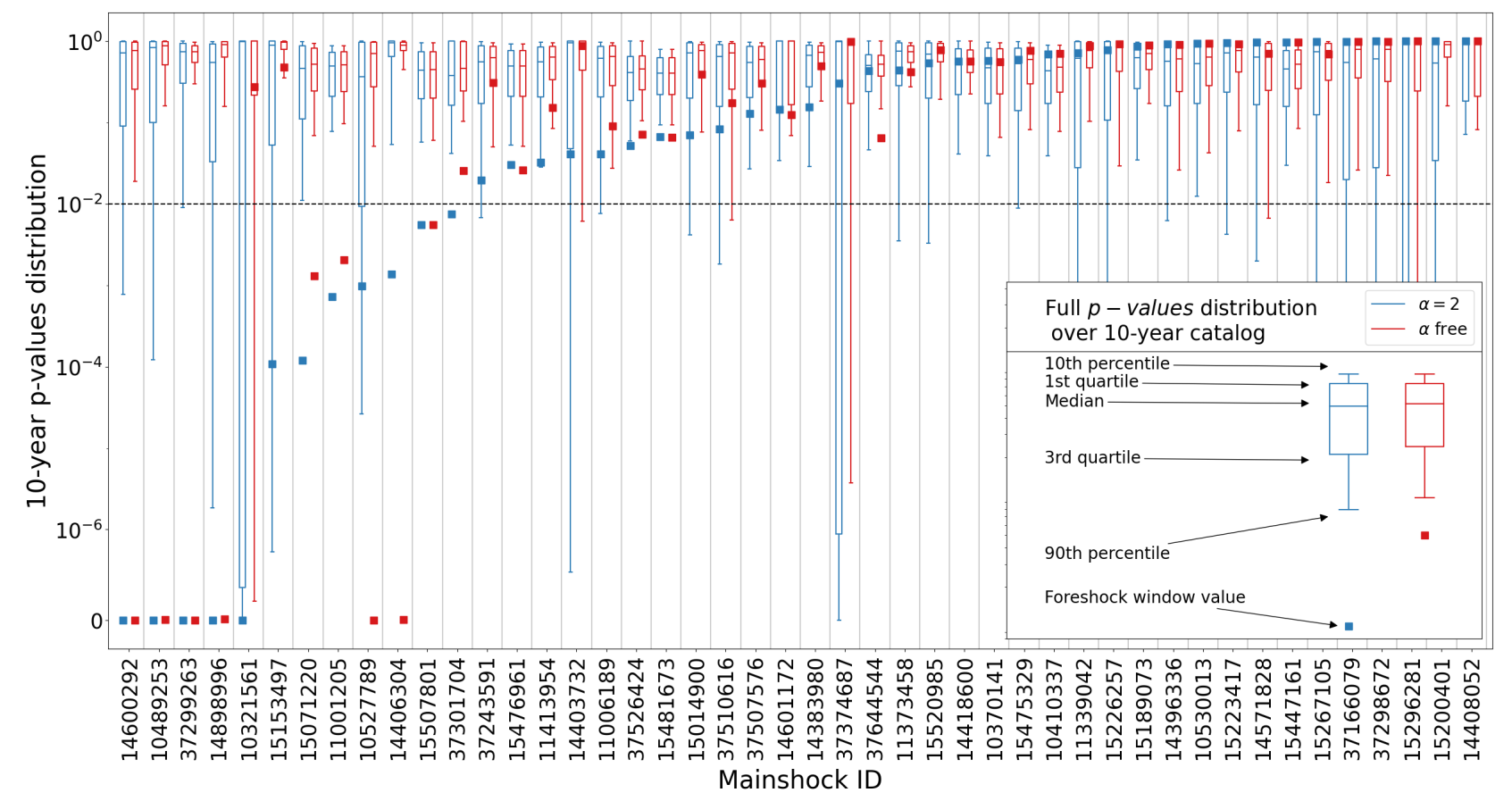

Figure S7. [QTM 9.5 dev] Same as S2 but this time using the Trugman and Ross (2019) mainshock selection from the QTM 9.5 dev catalog. We here find that 9 out of 46 mainshocks have anomalously high foreshock activity. 


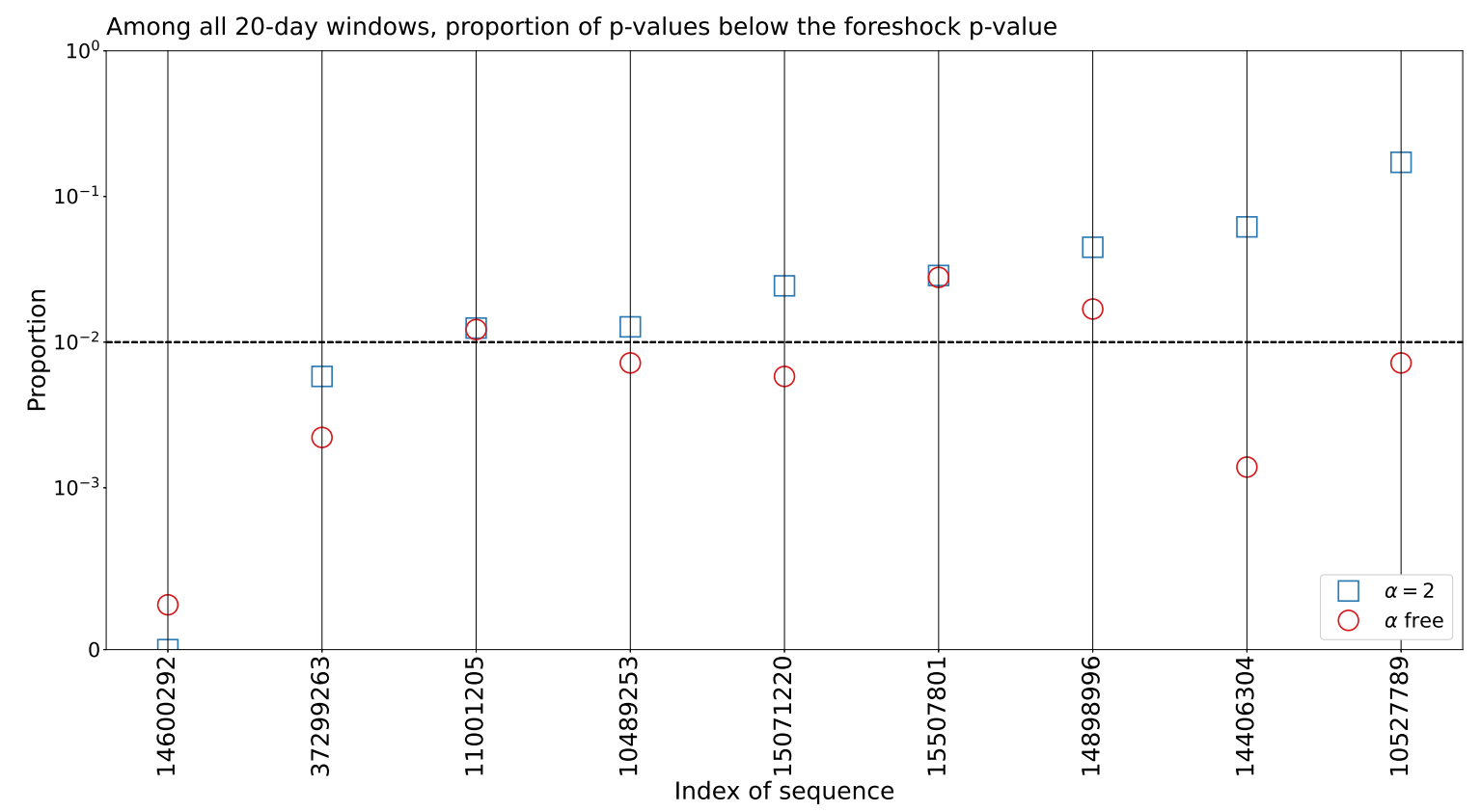

Figure S8. [QTM $9.5 \mathrm{dev}]$ Among all 20-day windows over 10 years, proportion $\hat{p}$ of windows with a p-value lower or equal to the 20-day foreshock window pvalue. The proportion $\hat{p}$ is computed for the 9 mainshocks with anomalously high foreshock activity and for the two ETAS estimates. We consider an anomalously high foreshock activity as mainshock-specific if $\hat{p}$ is below 0.01 for both estimates. Here, two foreshock anomalies are considered as mainshock-specific. 

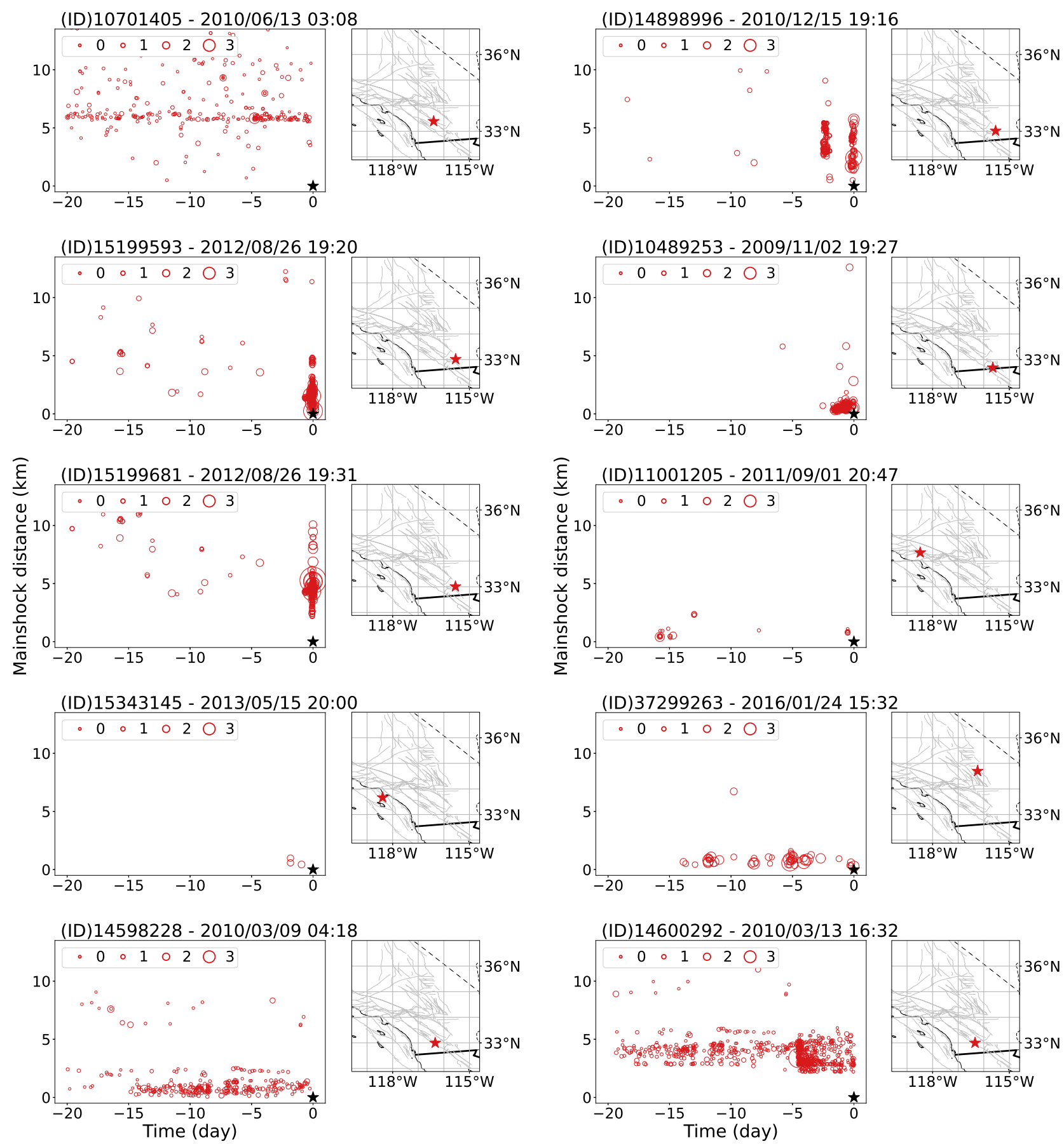

Figure S9. The 10 instances of anomalously high 20-day foreshock activity detected in this study. The mainshock distance correspond to the 3D distance in $\mathrm{km}$ (latitude, longitude and depth) between foreshocks and the mainshock (Black star) positions. The inset locate the mainshock position in Southern Californary 16, 2021, 12:43pm 


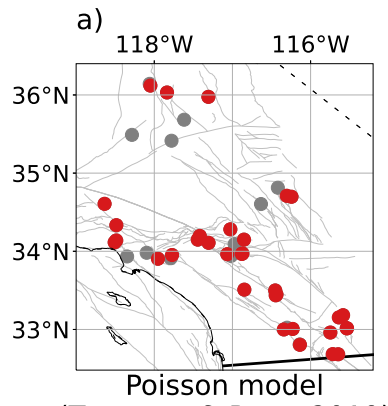

(Trugman \& Ross, 2019)

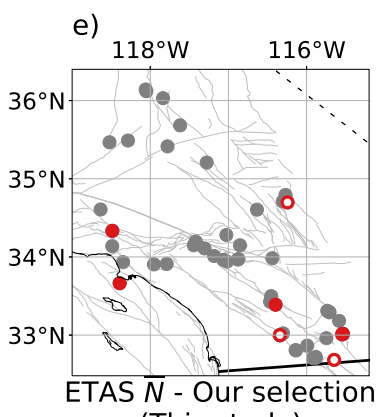

(This study) b)

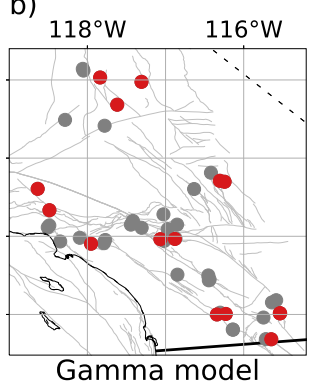

(van den Ende \& Ampuero 2020)

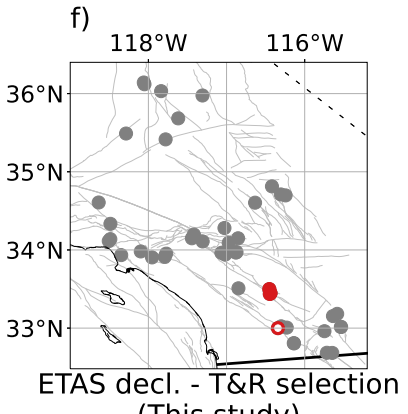

(This study)

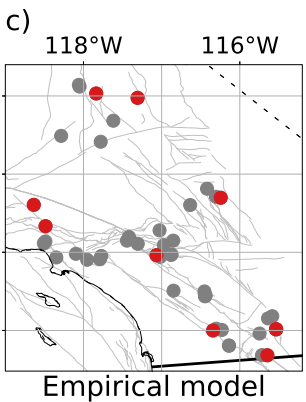

Ende \& Ampuero, 2020)

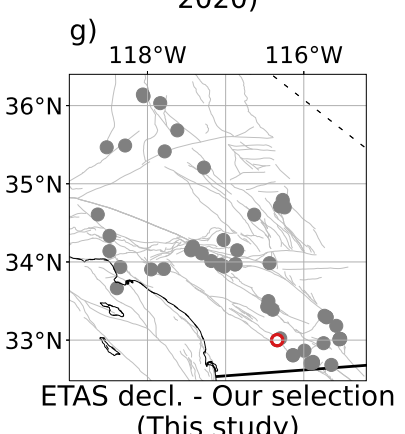

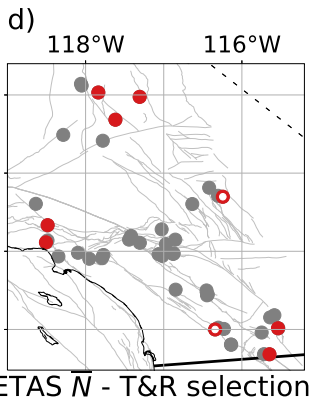

(This study)

Figure S10. Location of the mainshocks for all the analyses discussed in this study. The red locations are the mainshocks detected with a anomalously high 20-day foreshock activity $(p<0.01$, according to the model used). Red markers with a white inner core correspond to the anomalously high activity considered as mainshock specific in this study. (a) Poisson analysis of Trugman and Ross (2019). (b) Gamma analysis of van den Ende and Ampuero (2020). (c) Empirical analysis of van den Ende and Ampuero (2020). (d) This study ETAS expected 20day seismicity analysis on the Trugman and Ross (2019) mainshock selection from the QTM 9.5 dev catalog. (e) This study ETAS expected 20-day seismicity analysis on our own mainshock selection from the QTM 12.5 dev catalog. (f) This study ETAS declustering analysis on the Trugman and Ross (2019) mainshock selection from the QTM 9.5 dev catalog. (g) This study ETAS declustering analysis on our own mainshock selection from the QTM 12.5 dev catalog. Note that mainshocks with similar locations may appear superimposed.

February 16, 2021, 12:43pm 